\title{
Exponential Stabilization of Driftless Nonlinear Control Systems Using Homogeneous Feedback
}

\author{
Robert T. M'Closkey, Member, IEEE, and Richard M. Murray, Member, IEEE
}

\begin{abstract}
This paper focuses on the problem of exponential stabilization of controllable, driftless systems using time-varying, homogeneous feedback. The analysis is performed with respect to a homogeneous norm in a nonstandard dilation that is compatible with the algebraic structure of the control Lie algebra. It can be shown that any continuous, time-varying controller that achieves exponential stability relative to the Euclidean norm is necessarily non-Lipschitz. Despite these restrictions, we provide a set of constructive, sufficient conditions for extending smooth, asymptotic stabilizers to homogeneous, exponential stabilizers. The modified feedbacks are everywhere continuous, smooth away from the origin, and can be extended to a large class of systems with torque inputs. The feedback laws are applied to an experimental mobile robot and show significant improvement in convergence rate over smooth stabilizers.
\end{abstract}

Index Terms-Dilation, driftless, exponential stabilization, homogeneous, nonholonomic.

\section{INTRODUCTION}

$\mathbf{I}$ $\mathrm{N}$ this paper we consider the stabilization problem for driftless control systems of the form

$$
\dot{x}=X_{1}(x) u_{1}+\cdots+X_{m}(x) u_{m}, \quad x \in \mathbb{R}^{n} .
$$

We assume that the vector fields $X_{i}$ are analytic on $\mathbb{R}^{n}$ and that they are pointwise linearly independent. We further assume that the system is completely controllable: given any two points $x_{0}$ and $x_{1}$ and a time $T>0$ there exists a control $u$ defined on the time interval $[0, T]$ which steers the system between $x_{0}$ and $x_{1}$. Controllability is easily checked using the Lie algebra rank condition for nonlinear control systems (see, for example, Nijmeijer and van der Schaft [45] or Isidori [24]).

Controllable, driftless control systems arise in the study of mechanical systems with symmetries and nonholonomic constraints and represent the dynamics consistent with the kinematic constraints placed on the system by the presence of conservation laws or constraints. Typically, the inputs for a driftless control system correspond to the velocities of a mechanical system. Although in practice one almost always controls forces and torques in a mechanical system instead of velocities, in many instances it is possible to extend controllers that prescribe velocities to controllers that command forces and torques. Hence, we initially focus our attention on the

Manuscript received May 19, 1995; revised August 16, 1996 and November 8, 1996. Recommended by Associate Editor, J.-B. Pomet.

R. T. M'Closkey is with the Mechanical and Aerospace Engineering Department, University of California, Los Angeles, CA 90025 USA.

R. M. Murray is with the Department of Mechanical Engineering, California Institute of Technology, Pasadena, CA 91125 USA.

Publisher Item Identifier S 0018-9286(97)03391-6. driftless case and indicate later how to extend controllers to allow more general inputs.

The stabilization problem for driftless systems represents a challenge for nonlinear control theory because the linearization of the system is not controllable. In fact, as shown by Brockett [9], for this class of systems there does not exist a smooth (or even continuous) control law of the form $u_{i}=\alpha_{i}(x)$ which asymptotically stabilizes the system to an equilibrium point. As such, one is forced to rely on the use of strongly nonlinear techniques to stabilize the system. Results on asymptotic stability typically rely on the use of discontinuous feedback, time-varying feedback, or a combination of the two.

In this paper we concentrate on the problem of exponential stabilization of driftless systems. The usual definition of exponential stability does not readily apply to this problem, and one must use a broader definition of exponential stability. The approach to exponential stabilization in this paper makes use of the theory of homogeneous systems with nonstandard dilations [16], [21]. Using and extending the tools available from that area, we show how to construct and analyze exponential stabilizers for this class of systems. The extra structure which is available through the use of homogeneous systems allows us to circumvent many of the problems normally associated with non-Lipschitz systems and provides complete theory for driftless systems.

The main direct applications of the work presented here are control of mobile robots and other robotic systems with nonholonomic constraints (see [41] for introductory theory and examples). However, the basic techniques which we develop here are more broadly applicable and have potential application in a number of areas, including power converters [30], underwater vehicles [15], and novel robotic mechanisms [58]. With these and other applications in mind, we have tried to present many of the results in a context in which they can be applied to other strongly nonlinear stabilization problems.

This paper is organized as follows. In Section II we give a short review of the literature on stabilization of driftless control systems. This review is intended to orient the reader who is new to the area and also to describe the context for the results presented in this paper. Section III reviews results for homogeneous systems. The properties of homogeneous systems form the basis of the analysis in this paper. Section IV presents a method of improving the convergence rate of a driftless system when a smooth stabilizing feedback is already known. The convergence rate with the modified feedbacks is a modified notion of exponential stability. This method is applied to an experimental mobile robot in Section V. 
Section VI shows how to extend the exponentially stabilizing feedbacks through a set of integrators.

Finally, we indicate how the results in this paper apply to more general nonlinear control systems and indicate some of the directions for future work. Preliminary versions of some of the results in this paper have appeared in [33]-[37] and [42]. Additional technical results related to this work, as well as a more detailed introduction to homogeneous control systems, can be found in [32].

\section{RELATED WORK}

There have been a number of papers published on stabilization of nonholonomic systems over the past four years. A survey of the field can be found in the recent papers by Sørdalen and Egeland [57] and Samson [54]. We concentrate here on work that is most directly related to the results presented in this paper.

The basic limitations in stabilization of driftless systems were given in a 1983 paper by Brockett [9], where it was shown, among other things, that driftless control systems could not be stabilized to a point using continuous, static state feedback (for a particularly nice proof, see the survey paper by Sontag [56]). In 1990, Samson presented a paper in which he showed how to asymptotically stabilize a mobile robot to a point using time-varying, smooth state feedback [53]. The use of time-varying feedback avoided the difficulties captured by Brockett's necessary condition. Motivated by these results, Coron proved in 1991 that all controllable driftless systems could be stabilized to an equilibrium point using smooth, periodic, time-varying feedback [11]. (This result also follows from Sontag's work on universal controls [55].)

Coron's result opened the door to constructive approaches for stabilizing a general class of driftless control systems. This first such result was presented by Pomet [47], who developed a synthesis technique based on Coron's proof, which held for a fairly general class of systems including, as special cases, mobile robots, and mobile robots towing trailers. This result was extended to the general case by Coron and Pomet [12]. Additional techniques were given by Teel et al. [59] for a special class of driftless systems in so-called chained form [43].

A second approach to stabilizing nonholonomic systems involved the use of discontinuous feedbacks. One of the early results in this area was given by Bloch et al. [8] and involves the use of piecewise analytic feedbacks for stabilizing a nonholonomic mechanical system to a point. Unlike much of the other work on nonholonomic systems, the approach proposed by Bloch et al. allowed the use of either velocity or torque inputs rather than just velocity inputs. Another discontinuous stabilization approach was given by Canudas de Wit and Sørdalen [14], who developed piecewise smooth controllers for a set of low dimensional examples. The main application of their results was to mobile robots and one of the features was that they could guarantee that the control was discontinuous at only a finite number of times. Sørdalen and Egeland extended these results to systems in chained form [57].
One of the advantages of the discontinuous stabilization approaches over the smooth, time-periodic feedbacks is that discontinuous stabilizers usually give exponential convergence or convergence in finite time. Extending his previous work, Coron showed it is possible to generate time-periodic feedbacks which gave finite-time convergence and were smooth everywhere except the origin [12]. These results imply that exponential stabilizers exist which are time-periodic and smooth away from the equilibrium point. The necessity of nondifferentiable feedbacks even in the time-varying case can be found, for example, in [44], and is based on a straightforward linearization argument. One of the contributions of the present paper is to show more precisely how to construct feedback controllers which give exponential rates of convergence and are smooth everywhere except at the origin.

The exponential stability results presented in this paper rely on the properties of homogeneous systems and build off of several previous results on stability of homogeneous systems. The basic tools for dilations and homogeneous functions and vector fields are given in the monograph by Goodman [16] (see also Bacciotti [4]). Hermes has considered the application of homogeneous systems in control theory and has developed approximations which generalize the usual linear approximation theorems [20], [22], [23]. One of the earliest researchers to use notions of homogeneity for feedback stabilization of nonlinear systems was Dayawansa (see [13] and references therein). The use of homogeneous structure in stabilization problems has also been considered by Kawski [25], [26], who presented results for low-dimensional control systems with drift and defined the notion of exponential stability which we make use of here. Other work on homogeneous control systems includes the work of Ancona [2], who developed the analog of Poincaré normal forms for homogeneous vector fields, Rosier [52] on converse Lyapunov results for autonomous systems, and Pomet and Samson [49], who have extended their results on smooth stabilization to give exponential stabilization using tools similar to those presented in this paper.

In addition to time-varying feedback and discontinuous feedback, there have been many other approaches proposed for stabilization of driftless systems. Conditions for stabilization to a submanifold were given by Bloch et al. [8] (see also Montgomery [38]). Maschke and van der Schaft have generated controllers for stabilization to a submanifold using a Hamiltonian framework [31]. Hybrid strategies, involving the use of both discontinuous and time-varying feedbacks have been proposed by Pomet et al. [50] using a combination of Pomet's time-varying controllers near the origin and discontinuous feedback far away from the origin, and also Oelen et al. [46], who presented stabilizers for systems in chained form. Sørdalen and Egeland [57] have given controllers which involve switches at discrete instants in time and smooth feedback between switches. A similar technique has been used by Kolmanovsky and McClamroch [28], who use a discreteevent supervisor to generate switchings for controllers which give finite time convergence. Sliding-mode controllers have been explored by Bloch and Drakunov [7], and results on adaptive stabilization have been given by Bastin and Campion [5]. The use of nonsmooth changes of coordinates followed 


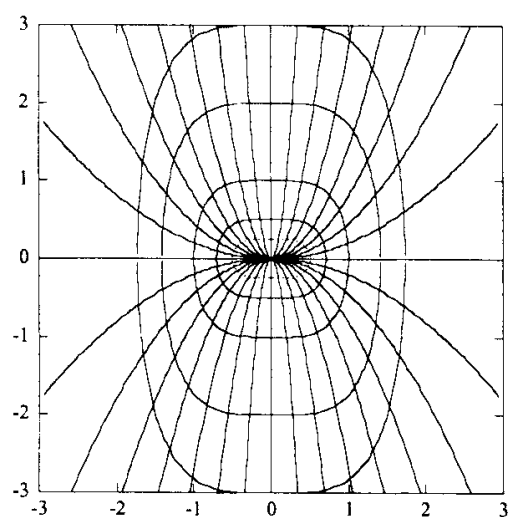

Fig. 1. Level sets of smooth homogeneous norm and trajectories of the Euler vector field $X_{E}$.

by smooth feedbacks in the transformed coordinates is a promising new direction which is being explored by Astolfi [3] and also Casalino et al. [10], [1].

Our own work in this area started with convergence analysis for the time-periodic, smooth controllers proposed by Pomet and Teel et al. These results showed that the controllers under consideration converged at a rate proportional to $1 / \sqrt{t}$ and hence gave very slow convergence. This motivated our work on exponential convergence and, based on the structure present in both chained and power form, we focused on the use of nonstandard dilations and homogeneous structure. Initial analysis tools were presented in [34], and preliminary results on controller synthesis were given in [37]. Experimental results on the application of various feedback control laws to a mobile robot system are described in detail in [33]. We have also derived a number of extensions to the basic work described in [37]. In [36] we describe how to extend exponentially stabilizing controllers which command the velocity to exponential controllers using torque inputs. In [42] we describe how to convert smooth, time-periodic, asymptotic stabilizers for driftless systems into exponential stabilizers which are smooth everywhere except the origin.

\section{HoMOGENEOUS SYSTEMS}

We now introduce some background material. To establish notation, functions will be denoted by lowercase letters and vector fields by capital letters. We will occasionally abuse notation and define the differential equation $\dot{x}=X(t, x)$ in local coordinates on $\mathbb{R}^{n}$ associated with the vector field $X$. The flow of a differential equation is denoted $\phi$, where $\phi\left(t, t_{0}, x_{0}\right)$ is the solution, at time $t$, which passes through the point $x_{0}$ at time $t_{0}$. When it is necessary to distinguish between flows of autonomous vector fields a superscript will be used, i.e., $\phi_{t}^{X}$ is the flow of $X, \phi_{t}^{Y}$ is the flow of $Y$, etc.

\section{A. Some Definitions}

This section reviews dilations and homogeneous vector fields. A dilation $\Delta_{\lambda}^{r}: \mathbb{R}^{n} \times \mathbb{R}^{+} \rightarrow \mathbb{R}^{n}$ is defined with respect to a fixed choice of coordinates $x=\left(x_{1}, x_{2}, \cdots, x_{n}\right)$ on $\mathbb{R}^{n}$ by assigning $n$ positive rationals $r=\left(r_{1}=1 \leq r_{2} \leq \cdots \leq r_{n}\right)$ and a positive real parameter $\lambda>0$ such that

$$
\Delta_{\lambda}^{r} x=\left(\lambda^{r_{1}} x_{1}, \cdots, \lambda^{r_{n}} x_{n}\right), \quad \lambda>0 .
$$

We usually write $\Delta_{\lambda}$ in place of $\Delta_{\lambda}^{r}$.

Definition 1: A continuous function $f: \mathbb{R} \times \mathbb{R}^{n} \rightarrow \mathbb{R}$ is homogeneous of degree $l \geq 0$ with respect to $\Delta_{\lambda}$ if $f\left(t, \Delta_{\lambda} x\right)=\lambda^{l} f(t, x)$.

Definition 2: A continuous vector field $X(t, x)=$ $\sum a_{i}(t, x) \partial / \partial x_{i}$ on $\mathbb{R} \times \mathbb{R}^{n}$ is homogeneous of degree $m \leq r_{n}$ with respect to $\Delta_{\lambda}$ if $a_{i}$ is degree $r_{i}-m$ for $i=1, \cdots, m$.

The variable $t$ represents explicit time dependence and is never scaled in our applications.

Definition 3: A continuous map from $\mathbb{R}^{n}$ to $\mathbb{R}, x \mapsto \rho(x)$ is called a homogeneous norm with respect to the dilation $\Delta_{\lambda}$ when:

1) $\rho(x) \geq 0, \quad \rho(x)=0 \Longleftrightarrow x=0$;

2) $\rho\left(\Delta_{\lambda} x\right)=\lambda \rho(x) \quad \forall \lambda>0$.

The homogeneous norm is called smooth when it is smooth on $\mathbb{R}^{n} \backslash\{0\}$.

For example, a smooth homogeneous norm may always be defined as

$$
\rho(x)=\left|x_{1}^{c / r_{1}}+x_{2}^{c / r_{2}}+\cdots+x_{n}^{c / r_{n z}}\right|^{1 / c}
$$

where $c$ is some positive integer evenly divisible by $r_{i}$. We are primarily interested in the convergence of time-dependent functions using a homogeneous norm as a measure of their size. When a vector field is homogeneous, it is most natural to use a corresponding homogeneous norm as the metric. The usual vector $p$-norms are homogeneous with respect to the standard dilation $\left(r_{i}=1\right)$.

Definition 4: The $\Delta$-sphere is defined as the set

$$
S_{\Delta}=\{x \mid \rho(x)=1\}
$$

where $\rho$ is a smooth homogeneous norm corresponding to the dilation $\Delta_{\lambda}$.

Definition 5: The Euler vector field corresponding to a dilation $\Delta_{\lambda}$ is defined as

$$
X_{E}(x)=\sum r_{i} x_{i} \frac{\partial}{\partial x_{i}} .
$$

Thus the images of trajectories of the system $\dot{x}=X_{E}(x)$ are the rays obtained by scaling the points on the sphere $S_{\Delta}$ with the dilation. Fig. 1 shows the level sets of the smooth homogeneous norm $\rho=\left(x_{1}^{4}+x_{2}^{2}\right)^{1 / 4}$ and the trajectories of the Euler vector field corresponding to the dilation $\Delta_{\lambda}(x)=$ $\left(\lambda x_{1}, \lambda^{2} x_{2}\right)$.

It is also possible to define homogeneous functions and vector fields in a more geometric fashion. The basic idea is to replace the Euler vector field with a smooth, asymptotically unstable vector field which defines the homogeneous rays of the system. Thus, given a smooth, complete vector field $Z$ on a manifold $M$ such that the flow of $-Z$ is asymptotically stable to the origin, we define a function $f: M \rightarrow \mathbb{R}$ to be homogeneous of degree $l \geq 0$ relative to $Z$ if

$$
L_{Z} f=l f \text {. }
$$


Similarly, a vector field $X: M \rightarrow T M$ is homogeneous of degree $m$ relative to $Z$ if

$$
[X, Z]=m X \text {. }
$$

Additional properties can be found in a recent paper by Kawski [27] and the references therein. Extension to the case where the degree of a function or vector field is not required to be constant has been considered by Praly [51].

\section{B. Homogeneous Approximations of Vector Fields}

This section reviews homogeneous approximations of sets of vector fields. The vector fields are the input vector fields of the controllable driftless system

$$
\dot{x}=X_{1}(x) u_{1}+\cdots+X_{m}(x) u_{m}
$$

The entire analysis is local, so we assume that vector fields are defined on $\mathbb{R}^{n}$. Furthermore, the vector fields are taken to be analytic. We are interested in obtaining an approximation, in the sense described below, of the set of vector fields $\left\{X_{1}, \cdots, X_{m}\right\}$. The Lie bracket of vector fields is $[\cdot, \cdot]$.

Let $\mathcal{L}\left(X_{1}, \cdots, X_{m}\right)$ be the Lie algebra generated by the set $\left\{X_{1}, \cdots, X_{m}\right\}$. The following definition specifies a special filtration of the Lie algebra of a finite set of generating vector fields.

Definition 6: The control filtration, $\mathcal{F}^{X}$, of $\mathcal{L}\left(X_{1}, \cdots, X_{m}\right)$ is a sequence of subspaces defined as

$$
\begin{aligned}
& \mathcal{F}_{0}^{\mathrm{X}}=\{0\} \\
& \mathcal{F}_{1}^{X}=\operatorname{span}\left\{X_{1}, \cdots, X_{m}\right\} \\
& \mathcal{F}_{2}^{X}=\operatorname{span}\left\{X_{1}, \cdots, X_{m},\left[X_{1}, X_{2}\right], \cdots,\left[X_{1}, X_{m}\right], \cdots\right. \\
& \left.\cdots,\left[X_{m-1}, X_{m}\right]\right\} \\
& \mathcal{F}_{k}^{X}=\operatorname{span}\{\text { all products of i-tuples from } \\
& \left.\left\{X_{1}, \cdots, X_{m}\right\} \text {, for } i \leq k\right\}
\end{aligned}
$$

and $\mathcal{F}^{X}=\left\{\mathcal{F}_{j}^{X}\right\}_{j \geq 0}$.

The set of vector fields is approximated about a specific point, $x_{0} \in \mathbb{R}^{n}$. This point is the desired equilibrium point in the sequel. Now let $F_{i}\left(x_{0}\right)$ be the subspace of $\mathbb{R}^{n}$ spanned by $Z\left(x_{0}\right)$, where $Z \in \mathcal{F}_{i}^{X}$. This yields an increasing sequence of vector subspaces

$$
\{0\}=F_{0}\left(x_{0}\right) \subset F_{1}\left(x_{0}\right) \subset \cdots \subset F_{i}\left(x_{0}\right) \subset \cdots \subset \mathbb{R}^{n} .
$$

This sequence must be stationary after some integer since it is assumed that the Lie algebra has full rank at $x_{0}$. In other words, since (2) is controllable $\operatorname{dim} F_{k}\left(x_{0}\right)=n$ for all $k$ greater than some minimal integer $N$. Now we count the growth in the dimension of the subspaces and set $n_{1}=$ $\operatorname{dim} F_{1}\left(x_{0}\right), n_{2}=\operatorname{dim} F_{2}\left(x_{0}\right), \cdots, n_{N}=n=\operatorname{dim} F_{N}\left(x_{0}\right)$. The following dilation is defined.
Definition 7: The dilation adapted to the filtration (at the point $x_{0}$ ) is the map

$$
\Delta_{\lambda}^{r} x=\left(\lambda^{r_{1}} x_{1}, \cdots, \lambda^{r_{n}} x_{n}\right)
$$

where the scalings satisfy $r_{i}=1$ for $1 \leq i \leq n_{1}, r_{i}=2$ for $n_{1}+1 \leq i \leq n_{2}$, etc.

Henceforth, in order to simplify the notation in the expressions to follow, it is assumed that $x_{0}=0$. This is achieved with a translation of the origin of the coordinate system.

Definition 8: The local coordinates adapted to the filtration $\mathcal{F}^{X}$ (denoted by $y$ ) are related to the original coordinates (denoted by $x$ ) by the local analytic diffeomorphism derived from composing flows of vector fields from the filtration

$$
x=\Phi(y)=\phi_{y_{1}}^{X_{\pi_{1}}} \circ \phi_{y_{2}}^{X_{\pi_{2}}} \circ \cdots \circ \phi_{y_{n}}^{X_{\pi_{2}}}(0)
$$

where $\phi_{t}^{X}\left(x_{0}\right)=\phi\left(t, 0, x_{0}\right)$ denotes the flow of the vector field $X$ and:
a) $X_{\pi_{i}} \in \mathcal{F}_{j}^{X}$ for $n_{j-1}+1 \leq i \leq n_{j}$;
b) $\operatorname{dim}\left\{X_{\pi_{1}}, \cdots, X_{\pi_{n}}\right\}=n$.

A vector field written in a local coordinate system will explicitly show the dependence, i.e., $X(x)$ is written in $x$ coordinates, while $X(y)$ is the same vector field written in $y$-coordinates. The importance of the local coordinates adapted to $\mathcal{F}^{X}$ is explained by the following theorem.

Theorem 1 [22, Th. 2.1]: Let $\mathcal{L}$ be a Lie algebra of vector fields on $\mathbb{R}^{n}$ and $\mathcal{F}=\left\{\mathcal{F}_{j}\right\}_{j \geq 0}$, an increasing filtration of $\mathcal{L}$ at zero with $\Delta_{\lambda}$ the dilation adapted to $\mathcal{F}$, and $y$ the local coordinates adapted to $\mathcal{F}$. If $X \in \mathcal{F}_{l}$, then

$$
X(y)=X^{l}(y)+X^{l-1}(y)+X^{l-2}(y)+\cdots
$$

where $X^{j}(y)$ is a vector field homogeneous of degree $j$ with respect to $\Delta_{\lambda}^{r}$.

In other words, if $X(y) \in \mathcal{F}_{l}$ is expanded in terms of vector fields which are homogeneous with respect to $\Delta_{\lambda}$, $X(y)=\sum_{j=r_{n}}^{-\infty} X^{j}(y)$, then $X^{r_{n}}(y)=\cdots=X^{l+1}(y)=0$ and the "leading order" vector field, $X^{l}(y)$, is degree $l$ with respect to $\Delta_{\lambda}$. This leading order vector field is termed the $\mathcal{F}$-approximation of $X \in \mathcal{F}_{l}$ in the $\mathcal{F}$-adapted coordinates. A useful property of the $\mathcal{F}$-approximation is given by the following proposition.

Proposition 1 [22, Corollary 2.2.1]: Let $\mathcal{F}=\left\{\mathcal{F}_{j}^{X}\right\}$ be the control filtration of $\mathcal{L}\left(X_{1}, \cdots, X_{m}\right)$ and $\left\{\mathcal{F}_{j}^{Y}\right\}_{j \geq 0}$ be the equivalently defined filtration of $\mathcal{L}\left(Y_{1}, \cdots, Y_{m}\right)$, where $Y_{i}$ is the $\mathcal{F}$-approximation of $X_{i}, i=1, \cdots, m$. Furthermore, let $F_{l}^{X}$ and $F_{l}^{Y}$ be the corresponding increasing sequence of vector subspaces of $\mathbb{R}^{n}$. Then

$$
F_{l}^{X}(0)=F_{l}^{Y}(0), \quad l=0,1, \cdots .
$$

Theorem 1 and Proposition 1 are very important for analytic driftless control systems: Theorem 1 guarantees that a degreeone approximation always exists if the original system is controllable, and Proposition 1 implies that the degree-one approximation is itself controllable. Thus, for purposes of control, these approximations are the correct ones to take (not the Jacobian linearization). When synthesizing feedbacks for driftless systems we will take advantage of the structure of the approximation. 
Remark 1: Bellaïche et al. [6] have defined the notion of local order, which they use to give the approximation a more intrinsic meaning. Their approximation coincides with the $\mathcal{F}$ approximation when the vector fields are written in local coordinates adapted to $\mathcal{F}$.

\section{Stability Definitions}

A modified definition of exponential stability is given below. The point $x=0$ is taken to be an equilibrium point of the differential equation $\dot{x}=X(t, x)$. For vectors $\|\cdot\|$ denotes the Euclidean norm, and for matrices it denotes the induced 2-norm.

The concept of exponential stability of a vector field is now defined in the context of a homogeneous norm. This definition was introduced by Kawski [26].

Definition 9: The equilibrium point $x=0$ is locally exponentially stable with respect to the homogeneous norm $\rho(\cdot)$ if there exist two constants $\alpha, \beta>0$ and a neighborhood of the origin $U$ such that

$$
\rho\left(\phi\left(t, t_{0}, x_{0}\right)\right) \leq \beta \rho\left(x_{0}\right) e^{-\alpha\left(t-t_{0}\right)} \quad \forall t \geq t_{0}, \forall x_{0} \in U .
$$

This stability type is denoted $\rho$-exponential stability to distinguish it from the usual definition of exponential stability.

This notion of stability is important when considering vector fields which are homogeneous with respect to a dilation. This definition is not equivalent to the usual definition of exponential stability except when the dilation is the standard dilation. This is evident from the following bounds on the Euclidean norm in terms of the smooth homogeneous norm given in (1) on the unit cube $\mathcal{C}=\left\{x:\left|x_{i}\right|<1, i=1, \cdots, n\right\}$ [recall $c \geq 2$ in Definition (1)]:

$$
\rho^{c / 2}(x) \leq\|x\| \leq M \rho(x) \text { for some } \quad M>0, x \in \mathcal{C}
$$

for some $M>0$. Hence, the solutions of a $\rho$-exponentially stable system which remain in the unit cube also satisfy

$$
\left\|\phi\left(t, t_{0}, x_{0}\right)\right\| \leq \beta M\left\|x_{0}\right\|^{\frac{2}{c}} e^{-\alpha\left(t-t_{0}\right)} .
$$

Thus, each state may be bounded by a decaying exponential envelope except that the size of the envelope does not scale linearly in the initial condition as in the usual definition of exponential stability. Exponential stability with respect to $\rho$ allows for non-Lipschitz dependence on the initial conditions. The expression in (5) will in general depend upon the particular coordinate system. However, the form of the bound in (6) remains the same under smooth diffeomorphism. It is useful to view the relation in (6) as a broader notion of exponential stability and $\rho$-exponential stability as a special case.

\section{Properties of Homogeneous Degree-Zero Vector Fields}

Some useful facts concerning degree-zero vector fields are reviewed in this section. A homogeneous degree-zero vector field $X(t, x)$ is invariant with respect to the dilation since

$$
\left(\Delta_{\lambda}\right)_{*} X(t, x)=X\left(t, \Delta_{\lambda} x\right), \quad \forall \lambda>0 .
$$

Thus, solutions scale to solutions with the dilation $\Delta_{\lambda} \phi\left(t, t_{0}, x_{0}\right)=\phi\left(t, t_{0}, \Delta_{\lambda} x_{0}\right)$. Some other properties are specified in the lemma below. Let $\pi$ denote the projection onto the homogeneous sphere $S_{\Delta}^{(n-1)}$ embedded in $\mathbb{R}^{n}, \pi: \mathbb{R}^{n} \backslash\{0\} \rightarrow S_{\Delta}^{n-1}$

$$
\pi(x)=\left(\frac{x_{1}}{\rho^{r_{1}}(x)}, \cdots, \frac{x_{n}}{\rho^{r_{n}}(x)}\right) .
$$

Lemma 1: Let $X(t, x)$ be a homogeneous degree-zero vector field. Then:

1) $X$ is $\pi$-related to a vector field $Y$ defined on $S_{\Delta}^{(n-1)}$, i.e., $\pi_{*} X=Y \circ \pi$;

2) uniform asymptotic stability is equivalent to global $\rho$ exponential stability.

The proof is a simple extension of Hahn's results [19].

\section{E. Uniqueness of Solutions}

Uniqueness of solutions gives a precise mathematical interpretation of the physical concept of determinism. The $\rho$-exponentially stabilizing feedbacks derived in the sequel are non-Lipschitz. We establish some sufficient conditions that the feedbacks must satisfy in order to guarantee unique solutions of the closed-loop system.

A homogeneous vector field is completely specified by the values assumed on the set $\{x: \rho(x)=1\}$ so any smoothness imposed on the vector field here is automatically extended to $\mathbb{R}^{n} \backslash\{0\}$ via the dilation. We will assume that the vector field is locally Lipschitz on $\mathbb{R}^{n} \backslash\{0\}$, i.e., for every $x \in \mathbb{R}^{n} \backslash\{0\}$ there exists a neighborhood of $x$ and some $0<L<\infty$ such that the vector field satisfies $\|X(t, y)-X(t, z)\| \leq L\|y-z\|$ for all $y$ and $z$ in this neighborhood. However, this does not imply that the vector field is Lipschitz in any neighborhood containing the origin. Degree-zero vector fields are of interest, so we concentrate on this case.

Lemma 2: Suppose $X(t, x): \mathbb{R} \times \mathbb{R}^{n} \rightarrow \mathbb{R}^{n}$ is a continuous homogeneous degree-zero vector field with respect to the dilation $\Delta_{\lambda}$, uniformly bounded with respect to $t$, and $x=0$ is an isolated equilibrium point. Furthermore, suppose that $X$ is locally Lipschitz everywhere except $x=0$. Then the flow of $X$ is unique.

Proof: The point $x=0$ is the only point where uniqueness may fail since $X$ is not necessarily Lipschitz there. However, no solution through a point $p \neq 0$ can reach the origin in finite time because this implies that $\rho\left(\phi\left(t, t_{0}, p\right)=0\right.$ in finite time. This is not possible since the equation describing the evolution of $\rho$ may be expressed as $\dot{\rho}=Q(t, y) \rho$, where $Q$ is a continuous function of $y$ and uniformly bounded in $t$. The point $y$ evolves on a compact set so there always exists a bound

$$
m \doteq \sup _{(t, y)}\|Q(t, y)\|
$$

The following inequalities on $\rho$ hold as a result of the bound on $Q$ :

$$
c_{1} e^{-m\left(t-t_{0}\right)} \leq \rho(x(t)) \leq c_{2} e^{m\left(t-t_{0}\right)}, \quad t \geq t_{0}
$$

where the $c_{i}$ 's are positive constants. Similarly, a solution cannot leave the origin in finite time. If this were possible, then the time reversed vector field (which has the same bounds on 
$\rho(x(t))$ as its forward time counterpart) has a solution which reaches the origin in finite time. This contradicts the above result. Thus solutions cannot leave or reach the origin in finite time, and so $x(t)=0$ for all $t$ is the only solution passing through the origin.

\section{F. Lyapunov Functions for Homogeneous Degree-Zero Vector Fields}

This section reviews converse Lyapunov stability theory for homogeneous systems and gives an extension for degreezero periodic vector fields. These results are important since the feedbacks derived in this paper exponentially stabilize an approximation of the driftless system, and the higher order (with respect to a dilation) terms neglected in the approximation process are shown to not locally change the stability of the system. The main theorem by Rosier in [52] states that given an autonomous continuous homogeneous vector field $\dot{x}=f(x)$ with asymptotically stable equilibrium point $x=0$, there exists a homogeneous Lyapunov function smooth on $\mathbb{R}^{n} \backslash\{0\}$ and differentiable as many times as desired at the origin. His theorem has been extended to timeperiodic degree zero systems in [48] and [32]. The extension is stated below without proof.

Theorem 2: Suppose the differential equation $\dot{x}=X(t, x)$ satisfies the following properties.

1) $X$ is continuous in $t$ and $x$.

2) $X(t, 0)=0$ for all $t$.

3) $X(t+T, x)=X(t, x)$ for all $x \in \mathbb{R}^{n}$.

4) $X$ is homogeneous degree zero (in $x$ ) with respect to the dilation $\Delta_{\lambda}=\left(\lambda^{r_{1}} x_{1}, \cdots, \lambda^{r_{n}} x_{n}\right)$.

5) The solution $x(t)=0$ is asymptotically stable.

Let $k$ be a positive integer. Then there exists a function $\bar{V}: \mathbb{R} \times \mathbb{R}^{n} \rightarrow \mathbb{R}$ such that:

- $\bar{V}(t, x)$ is smooth for $x \in \mathbb{R}^{n} \backslash\{0\}$;

- $\bar{V}(t, 0)=0, \bar{V}(t, x)>0$ when $x \neq 0$;

- $\bar{V}$ is degree $k$ with respect to $\Delta_{\lambda}$, i.e., $\bar{V}\left(t, \Delta_{\lambda} x\right)=$ $\lambda^{k} \bar{V}(t, x)$

- $\bar{V}(t+T, x)=\bar{V}(t, x)$ for all $x \in \mathbb{R}^{n}$ and smooth with respect to $t$

- $\frac{d \bar{V}}{d t}(t, x)=\frac{\partial \bar{V}}{\partial t}(t, x)+\nabla \bar{V}(t, x) \cdot X(t, x)<0$ for all $x \neq 0$.

Finally, the following proposition concerning the stability of perturbed degree-zero vector fields concludes this section. The proof is elementary and follows the time-invariant case in [52].

Proposition 2: Let $x=0$ be an asymptotically stable equilibrium point of the $T$-periodic continuous homogeneous degree-zero vector field $\dot{x}=X(t, x)$. Consider the perturbed system

$$
\dot{x}=X(t, x)+R(t, x) .
$$

Assume each component of $R(t, x)$ may be uniformly bounded by

$$
\left|R_{i}(t, x)\right| \leq m \rho^{r_{i}+1}(x) \quad i=1, \cdots, n, \quad x \in U
$$

where $U$ is an open neighborhood of the origin and $\rho(\cdot)$ is a homogeneous norm compatible with the dilation that leaves the unperturbed equation invariant. Then $x=0$ remains a locally exponentially stable equilibrium of the perturbed equation (7).

\section{SyNTHESIS METHODS}

We now consider how to obtain exponentially stabilizing feedbacks. The stability type is not the familiar exponential stability definition, but rather $\rho$-exponential stability. As pointed out in Section III-C, $\rho$-exponential stability can be locally recast into the bound $\left\|\phi\left(t, t_{0}, x_{0}\right)\right\|_{2} \leq$ $M\left\|x_{0}\right\|_{2}^{1 / \sigma} e^{-\alpha\left(t-t_{0}\right)} \quad$ for some $M>0, \alpha>0, \sigma>1$. Thus each state is bounded by a decaying exponential envelope, but the dependence on the initial condition is allowed to be more general than that in the usual definition of exponential stability. The standing assumption in the remainder of the paper is that (2) has been transformed to the adapted coordinates and that a degree-one homogeneous approximation has been computed.

\section{A. Limitations of Lipschitz Feedback}

The dilation associated with the input vector field approximations and feedbacks will always have $r_{n}>1$ since at least one level of Lie brackets is required to achieve controllability of the system. Thus the degree-one feedbacks which we shall construct in this section are not Lipschitz at the origin even though they may be locally Lipschitz on $\mathbb{R}^{n} \backslash\{0\}$. A natural question is to what extent non-Lipschitz feedback is necessary for exponential convergence. This question is answered within the context of standard exponential stability by the following theorem.

Theorem 3: Consider the control system $\dot{x}=X(x, u)$. Assume that $X$ is $C^{1}$ in both arguments and $X(0,0)=0$. If the linearization around $x=0$ and $u=0$ has an uncontrollable mode with real part of the eigenvalue equal to zero, then solutions of the closed-loop system with Lipschitz feedback $u(t, x), u(t, 0)=0$ do not satisfy the exponential stability bound $\left\|\phi\left(t, t_{0}, x_{0}\right)\right\| \leq \beta\left\|x_{0}\right\| e^{-\alpha\left(t-t_{0}\right)}$ for any $\alpha, \beta>0$.

A proof of this result using nonsmooth analysis can be found in [32]. A simpler proof, using more standard tools, has been developed independently by Gurvitz [17] who extends the ideas from [18] to systems with drift vector fields. The result in [18] states that an exponentially stabilizing (in the standard sense) feedback must be Hölder continuous with Hölder exponent equal to the inverse of number of Lie brackets required to achieve full rank in the control Lie algebra. Morin has also recently given a proof of Theorem 3 [39]. ${ }^{1}$ It is still an open question as to whether standard exponential stability can be achieved for driftless systems using non-Lipschitz feedback.

\section{B. From Asymptotic to Exponential Stabilizers}

Several researchers have either given explicit smooth controllers or constructive algorithms that produce smooth timeperiodic feedbacks which asymptotically stabilize mobile robot and satellite models [59], [47], [60]. A desirable aspect of these methods is the fact that, in many instances, the control laws can be written in terms of algebraic operations between simple functions. The implication of this fact should not be

\footnotetext{
${ }^{1}$ Added in print.
} 
underestimated since implementation of such a control law is straightforward. The algorithm in Theorem 4 gives sufficient conditions under which smooth asymptotically stabilizing feedbacks can be rescaled into homogeneous $\rho$-exponentially stabilizing feedbacks. The design procedure is systematic in the sense that if the conditions of the theorem are satisfied, then the homogeneous feedback may be computed directly from the original feedback. This algorithm is applied to the smooth feedback used for the mobile robot and results in an enormous improvement in convergence rate. A more direct method of computing $\rho$-exponential stabilizers, based on an extension of Pomet's original algorithm [47], is briefly noted at the end of this section.

Recall that the Euler vector field, $X_{E}(x)$, corresponding to this dilation is given by the equations $\dot{x}_{i}=r_{i} x_{i}, i=1, \cdots, n$. The following theorem specifies the conditions under which an asymptotic stabilizer can be modified into an exponential stabilizer. Most smooth stabilizing controllers are time-periodic, so we restrict ourselves to this case.

Theorem 4: Suppose the closed-loop driftless system $\dot{x}=$ $\sum_{i=1}^{m} X_{i}(x) u_{i}(t, x)$ satisfies the following conditions.

1) The input vector fields are homogeneous degree one with respect to $\Delta_{\lambda}$.

2) The feedbacks $u_{i}(t, x)$ are smooth, $T$-periodic, and asymptotically stabilize the origin.

3) There exists a smooth, positive definite, $T$-periodic function $V(t, x)$ such that $\frac{d}{d t} V(t, x)<0$ along nonzero solutions of the closed-loop system.

4) There exists $C>0$ such that the level sets parameterized by $t$

$$
G_{t}^{C}=\{x \mid V(t, x)=C\}
$$

are bounded and satisfy the following transversality condition with the Euler vector field:

$$
L_{X_{E}} V(t, x)>0, \quad x \in G_{t}^{C} .
$$

Under these conditions, the original feedbacks may be modified to the following $\rho$-exponentially stabilizing feedbacks:

$$
\tilde{u}_{i}(t, x)=\tilde{\rho}(t, x) u_{i}\left(t, \gamma_{t}(x)\right), \quad i=1, \cdots, m
$$

where $\tilde{\rho}: \mathbb{R} \times \mathbb{R}^{n} \rightarrow \mathbb{R}^{+}$is a uniquely defined homogeneous degree-one function such that

$$
\left.\widetilde{\rho}(t, x)\right|_{x \in G_{t}^{C}}=1
$$

and the map $\gamma_{t}: \mathbb{R}^{n} \backslash\{0\} \rightarrow G_{t}^{C}$ is defined as

$$
\gamma_{t}(x)=\Delta_{\lambda} x=\bar{x} \in G_{t}^{C}, \quad \text { for some } \lambda>0 \text {. }
$$

Remark 2: In many cases the stabilizing feedback is derived from Lyapunov analysis, and so the closed-loop system has a function which may be tested for the properties given in the theorem.
Remark 3: The function $\tilde{\rho}(t, x)$ is computed by searching over a single scalar parameter $\lambda$ such that $V\left(t, \Delta_{\lambda} x\right)=C$. While analytical expressions are unlikely to exist, there are circumstances in which $\tilde{\rho}$ is very easy to numerically compute. In the examples in the sequel, the transversality condition is satisfied in a neighborhood containing the origin and the set $G_{t}^{C}$. For fixed $t$ and $x, V\left(t, \Delta_{\lambda} x\right)$ is a monotone increasing function of $\lambda$ in this neighborhood so simple search methods may be employed to efficiently compute $\lambda$ and $\tilde{\rho}$. Alternatively, a stable ODE can be used to track $\lambda$, as pointed out by Praly [51].

Proof: We first show that $\tilde{\rho}$ and $\gamma_{t}$ are well defined quantities. Define the value of the function $g: \mathbb{R} \times \mathbb{R}^{n} \backslash\{0\} \rightarrow$ $\mathbb{R}^{+}$to be the $\lambda \in \mathbb{R}^{+}$which solves

$$
F(\lambda, t, x) \doteq V\left(t, \Delta_{\lambda} x\right)-C=0 \text {. }
$$

In other words, $g(t, x)$ returns the dilation scaling required to map the point $x \neq 0$ to the point $\bar{x} \in G_{t}$ on the same homogeneous ray at time $t$. We show the point $\bar{x}$ is unique. The transversality condition implies that the projection $\left.\pi\right|_{G_{t}^{C}}: G_{t}^{C} \rightarrow S_{\Delta}^{n-1}$ is a local diffeomorphism since the flow of $X_{E}$ may be used to define the diffeomorphism (trajectories of $X_{E}$ are transversal to $G_{t}^{C}$ and $S_{\Delta}^{n-1}$ ). The projection when restricted to $G_{t}^{C}$ is also one-to-one. This is established in the following way. Suppose there is more than one point in the pre-image $\pi^{-1}(x), x \in S_{\Delta}^{n-1}$, in the set $G_{t}^{C}$. Select two points and label the point with the smallest homogeneous norm as $x_{0}$ and the other point $x_{1}$. Flow along $X_{E}$ starting at $\left(t_{0}, x_{0}\right)$. For some $t_{1}>t_{0}$ the point $x_{1}$ is reached. Denote the flow of $X_{E}$ as $\phi_{t}(x)$. Since $L_{X_{E}} V>0$, then there is an $\epsilon>0$ such that $V\left(t, \phi_{t_{0}+\epsilon}\left(x_{0}\right)\right)>C$ and $V\left(t, \phi_{t_{1}-\epsilon}\left(x_{1}\right)\right)<C$. The Lyapunov function is continuous, so the intermediate value theorem establishes that there is a point, labeled $x_{2}$, such that $V\left(t, x_{2}\right)=C$ and $\rho\left(x_{0}\right)<\rho\left(x_{2}\right)<\rho\left(x_{1}\right)$. By hypothesis $L_{X_{E}} V\left(t, x_{2}\right)>0$ so we may proceed to show that there is a point, $x_{3}$, on the homogeneous ray between $x_{0}$ and $x_{2}$ such that $V\left(t, x_{3}\right)=C$. Continuing with this process, a bounded sequence $\left\{x_{k}\right\}_{k=0}^{\infty}$ is generated. However, in any neighborhood of an accumulation point of the sequence, the projection cannot be a local diffeomorphism. This contradicts the transversality assumption. Thus the point $\bar{x}$ is unique, and the projection is a global diffeomorphism between $G_{t}^{C}$ and $S_{\Delta}^{n-1}$ for each fixed $t$.

The map from $x$ to $\bar{x}$ is $\left(\left.\pi\right|_{G_{t}^{C}}\right)^{-1} \circ \pi$ and $g(t, x)=$ $\rho(\bar{x}) / \rho(x)$. The smoothness of $g$ is guaranteed by the implicit function theorem when $\partial F / \partial \lambda\left(t, \Delta_{\lambda} x\right) \neq 0$. Suppose that $(\lambda, t, x)$ satisfies $(8)$, then

$$
\begin{aligned}
\frac{\partial F}{\partial \lambda}\left(t, \Delta_{\lambda} x\right) & =\frac{\partial V}{\partial \lambda}\left(t, \Delta_{\lambda} x\right) \\
& =\sum_{i=1}^{n} \frac{\partial V}{\partial x_{i}}\left(t, \Delta_{\lambda} x\right) r_{i} \lambda^{r_{i}-1} x_{i} \\
& =\frac{1}{\lambda} \sum_{i=1}^{n} \frac{\partial V}{\partial x_{i}}\left(t, \Delta_{\lambda} x\right) r_{i} \lambda^{r_{i}} x_{i} \\
& =\frac{1}{\lambda} L_{X_{E}} V\left(t, \Delta_{\lambda} x\right) .
\end{aligned}
$$


The term $L_{X_{E}} V\left(t, \Delta_{\lambda} x\right)$ is precisely the transversality condition on the set $G_{t}^{C}$. Similarly, $\partial g / \partial x_{i}(t, x) \neq 0$ for all $x \in G_{t}^{C}$.

We now show that $g$ is degree -1 . If $g(t, x)=\lambda$, then $g\left(t, \Delta_{\sigma} x\right)$ is the $\lambda_{0}$ that solves $V\left(t, \Delta_{\lambda_{0}} \Delta_{\sigma} x\right)-C=0$. Since $\Delta_{\lambda_{0}} \Delta_{\sigma} x=\Delta_{\lambda_{0} \sigma} x$, then $\lambda=\lambda_{0} \sigma$ so $g\left(t, \Delta_{\sigma} x\right)=\lambda / \sigma=$ $g(t, x) / \sigma$.

The function $\gamma: \mathbb{R} \times \mathbb{R}^{n} \backslash\{0\} \rightarrow G_{t}^{c}$ is

$$
\gamma(t, x) \doteq \Delta_{g(t, x)} x
$$

Note that $\gamma\left(t, \Delta_{\lambda} x\right)=\gamma(t, x)$ for all $\lambda>0 . \tilde{\rho}: \mathbb{R} \times \mathbb{R}^{n} \rightarrow \mathbb{R}^{+}$ is defined as

$$
\tilde{\rho}(t, x) \doteq \begin{cases}\frac{1}{g(t, x)} & x \neq 0 \\ 0 & x=0\end{cases}
$$

Furthermore, for any $\bar{x} \in G_{t}^{c}, \tilde{\rho}(t, \bar{x})=1$ since $\gamma(t, \bar{x})=\bar{x}$. The definitions may be used to show that $\gamma(t, \cdot)$ is smooth on $\mathbb{R}^{n} \backslash\{0\}$ and $\tilde{\rho}(t, \cdot)$ is continuous on $\mathbb{R}^{n}$ and smooth on $\mathbb{R}^{n} \backslash\{0\}$. Furthermore, $\tilde{\rho}$ is homogeneous degree $1 . T$ periodicity of $\tilde{\rho}$ and $\gamma$ is evident from the fact that $V$ is $T$-periodic.

The modified feedbacks are defined as

$$
\tilde{u}_{i}(t, x) \doteq \tilde{\rho}(t, x) u_{i}(t, \gamma(t, x))
$$

These functions are degree one since

$$
\begin{aligned}
& \tilde{u}_{i}\left(t, \Delta_{\lambda} x\right)=\tilde{\rho}\left(t, \Delta_{\lambda} x\right) u_{i}\left(t, \gamma\left(t, \Delta_{\lambda} x\right)\right) \\
& =\lambda \tilde{\rho}(t, x) u_{i}(t, \gamma(t, x)) \\
& =\lambda \tilde{u}_{i}(t, x) .
\end{aligned}
$$

These functions agree with the original feedbacks on $G_{t}^{C}$; i.e., for $\bar{x} \in G_{t}^{C} \tilde{u}_{i}(t, \bar{x})=u_{i}(t, \bar{x})$. We now show that the closed-loop system with the newly defined feedbacks is $\rho$-exponentially stable. The closed-loop system with the new feedbacks is denoted $\dot{x}=\tilde{X}(t, x)$. The closed-loop system is degree zero since the feedback is degree one and the input vector fields are degree one. Hence, all we need to show is uniform asymptotic stability with the modified feedbacks. This is accomplished by using $\tilde{\rho}(t, x)$ as a Lyapunov function.

First we show that $\tilde{\rho}$ is positive definite and decrescent. The assumptions on $V(t, x)$ imply that there exist two continuous positive definite, strictly increasing functions, $\phi_{1}$ and $\phi_{2}$, such that $\phi_{1}(\|x\|) \leq V(t, x) \leq \phi_{2}(\|x\|)$ for all $x$ and $t$. Any $x \in G_{t}^{C}$ must satisfy the bounds $\phi_{2}^{-1}(C) \leq\|x\| \leq \phi_{1}^{-1}(C)$. Defining the constants

$$
c_{1}=\min _{\|x\|=\phi_{2}^{-1}(C)} \rho(x) \quad \text { and } \quad c_{2}=\max _{\|x\|=\phi_{1}^{-1}(C)} \rho(x)
$$

it is straightforward to verify that $\rho(x) / c_{2} \leq \tilde{\rho}(t, x) \leq$ $\rho(x) / c_{1}$ for all $x$ and $t$. Thus $\tilde{\rho}$ is positive definite and decrescent. Define the function $\tilde{V}(t, x)=\tilde{\rho}$. The time derivative of $\tilde{V}$ along nonzero solutions of the system with the feedback in (9) is

$$
\begin{aligned}
& \frac{d \tilde{V}}{d t}(t, x)=\left(\frac{d}{d t} \frac{1}{g}\right)(t, x) \\
&=-\frac{1}{g^{2}(t, x)}\left(\frac{\partial g}{\partial t}(t, x)+D_{x} g(t, x)(\tilde{X})\right)(t, x) \\
&=-\frac{1}{g^{2}(t, x)}\left(-\frac{g(t, x)}{L_{X_{E}} V(t, \bar{x})} \frac{\partial V}{\partial t}(t, \bar{x})-\frac{1}{L_{X_{E}} V(t, \bar{x})}\right. \\
&\left.\cdot \sum_{i=1}^{n} g^{r_{i}+1}(t, x) \frac{\partial V}{\partial x_{i}}(t, \bar{x}) \tilde{X}_{i}(t, x)\right)
\end{aligned}
$$

where $\bar{x}=\Delta_{g(t, x)} x \in G_{t}^{c}$

$$
\begin{aligned}
& =\frac{1}{g(t, x) L_{X_{E}} V(t, \bar{x})}\left(\frac{\partial V}{\partial t}(t, \bar{x})+\right. \\
& \left.\qquad \sum_{i=1}^{n} \frac{\partial V}{\partial x_{i}}(t, \bar{x}) \tilde{X}_{i}\left(t, \Delta_{g(t, x)} x\right)\right) \\
& =\frac{1}{g(t, x) L_{X_{E}} V(t, \bar{x})}\left(\frac{\partial V}{\partial t}(t, \bar{x})+\sum_{i=1}^{n} \frac{\partial V}{\partial x_{i}}(t, \bar{x}) X_{i}(t, \bar{x})\right) \\
& =\frac{\tilde{\rho}(t, x)}{L_{X_{E}} V(t, \bar{x})} \frac{d V}{d t}(t, \bar{x})<0 .
\end{aligned}
$$

$\rho$-exponential stability follows from the fact that the closedloop system is degree zero.

The new feedback is as smooth on $\mathbb{R}^{n} \backslash\{0\}$ as the original feedback restricted to the level set of the Lyapunov function in the proof of Theorem 4. The original feedback is assumed to be smooth, and so solutions of the closed-loop system with the modified feedback are unique by Lemma 2 .

Theorem 4 also applies to asymptotically stable systems when the Lyapunov function is only negative semi-definite along the closed-loop system trajectories. The following proposition addresses this situation.

Proposition 3: Consider a driftless system which satisfies Conditions 1), 2), and 4) of Theorem 4 and with Condition 3) replaced by:

3') there exists a smooth, positive definite, T-periodic function $V(t, x)$ such that $\frac{d}{d t} V(t, x) \leq 0$ along solutions of the closed-loop system.

The modified feedbacks

$$
\tilde{u}_{i}(t, x) \doteq \tilde{\rho}(t, x) u_{i}\left(t, \gamma_{t}(x)\right), \quad i=1, \cdots, m
$$

where $\tilde{\rho}$ and $\gamma_{t}$ are defined as in Theorem $4, \rho$-exponentially stabilizing the system.

Proof: Construct $\tilde{V}(t, x)$ as in the proof of Theorem 4 and recall

$$
\frac{d \tilde{V}}{d t}(t, x)=\frac{\tilde{\rho}(t, x)}{L_{X_{E}} V(t, \bar{x})} \frac{d V}{d t}(t, \bar{x}) \leq 0
$$

so the system with modified feedbacks is at least uniformly stable. We show that asymptotic stability of the system with the modified feedback $\tilde{u}_{i}(t, x), i=1, \cdots, m$ is implied by asymptotic stability of the system with the smooth feedback $u_{i}(t, x), i=1, \cdots, m$.

Suppose the system with feedback $\tilde{u}_{i}$ is not asymptotically stable. Then there exists a solution $x_{0}(t) \neq 0$ such that $\tilde{V}\left(t, x_{0}(t)\right)=\alpha$, for some $\alpha>0$ (see [19, Th. 55.1]). In 
fact, the dilation may be used to show that there is an entire family of solutions with the property $\tilde{V}(t, x)=$ constant. However, we are interested in only one particular solution corresponding to the level set $\tilde{V}=1$. Choose $\lambda$ such that $\alpha \lambda=1$ and define another solution of the closed-loop system as $\tilde{x}(t)=\Delta_{\lambda} x_{0}(t)$. Since

$$
\tilde{V}(t, \tilde{x}(t))=\tilde{V}\left(t, \Delta_{\lambda} x_{0}(t)\right)=\alpha \lambda=1
$$

then $\tilde{x}(t) \subset G_{t}^{C} \quad \forall t$. Thus, $u_{i}(t, \tilde{x}(t))=\tilde{u}_{i}(t, \tilde{x}(t))$ and $\tilde{x}(t)$ is a solution of the closed-loop system with the original smooth feedbacks. Furthermore, $V(t, \tilde{x}(t))=C$ (recall that $\{(t, x) \mid \tilde{V}(t, x)=1\}=\{(t, x) \mid V(t, x)=C\})$ so that the system with smooth feedbacks is not asymptotically stable.

We conclude this section by briefly mentioning another method to synthesize locally $\rho$-exponentially stabilizing controllers for a class of driftless systems. This method is an extension of Pomets's algorithm [47]. If the homogeneous approximation of the driftless system satisfies the rank condition

$$
\begin{aligned}
\operatorname{rank}\{ & X_{\pi_{1}}^{1}, X_{\pi_{2}}^{1}, \cdots, X_{\pi_{m}}^{1}, \\
& {\left[X_{\pi_{1}}^{1}, X_{\pi_{2}}^{1}\right], \cdots,\left[X_{\pi_{1}}^{1}, X_{\pi_{m}}^{1}\right], \cdots, } \\
& \left.\operatorname{ad}_{X_{\pi_{1}}^{1}}^{j} X_{\pi_{2}}^{1}, \cdots, \operatorname{ad}_{X_{\pi_{1}}^{1}}^{j} X_{\pi_{m}}^{1}, \cdots\right\}\left(x_{0}\right)=n
\end{aligned}
$$

for some permutation, $\pi$, of the set $\{1,2, \cdots, m\}$, then the steps in [47] may be modified to produce homogeneous feedbacks, smooth on $\mathbb{R}^{n} \backslash\{0\}$, which $\rho$-exponentially stabilize the approximating system [37], [32]. This extended method is appealing because it is easy to check the condition in (10). The drawback of this approach is that the feedbacks must be stored in look-up tables. This is not an attractive feature for real-time implementation since the number of points which must be computed and stored grows exponentially with $n$, the state dimension.

\section{Examples}

This section applies the algorithm from Theorem 4 to two smooth asymptotically stabilizing control laws designed for the prototype driftless system.

Example 1: Consider the three-dimensional two input driftless system

$$
\dot{x}_{1}=u_{1}, \quad \dot{x}_{2}=u_{2}, \quad \dot{x}_{3}=x_{2} u_{1} .
$$

This system is its own homogeneous degree-one $\mathcal{F}$ approximation. The dilation is $\Delta_{\lambda}(x)=\left(\lambda x_{1}, \lambda x_{2}, \lambda^{2} x_{3}\right)$. A smooth asymptotically stabilizing feedback for the system takes from [59] the functions

$$
\begin{aligned}
& u_{1}(t, x)=-x_{1}+x_{3} \cos t \\
& u_{2}(t, x)=-x_{2}+x_{3}^{2} \sin t .
\end{aligned}
$$

Asymptotic stability of the closed-loop system can be shown using the Lyapunov function

$$
\begin{aligned}
V(t, x)= & \left(x_{1}-\frac{x_{3}}{2}(\cos t+\sin t)\right)^{2}+ \\
& \left(x_{2}-\frac{x_{3}^{2}}{2}(\sin t-\cos t)\right)^{2}+x_{3}^{2} .
\end{aligned}
$$

Thus, we need to check the boundedness and transversality condition with a level set of the Lyapunov function. $V$ may be approximated by the quadratic form $\tilde{V}=\langle x, B x\rangle$ for $\|x\|$ sufficiently small, where

$$
B=\left[\begin{array}{ccc}
1 & 0 & -\frac{1}{2} \alpha \\
0 & 1 & 0 \\
-\frac{1}{2} \alpha & 0 & 1+\frac{1}{4} \alpha^{2}
\end{array}\right]
$$

and $\alpha=\cos t+\sin t \in[-\sqrt{2}, \sqrt{2}]$. Level sets of $\tilde{V}$ are bounded and imply that level sets of $V$ are bounded for $\|x\|$ sufficiently small (this property can be verified by direct calculation too). The inner product between the level sets of $\widetilde{V}$, and the Euler vector field is $L_{X_{E}} \tilde{V}=\langle x, \tilde{B} x\rangle$, where $\tilde{B}$ is the symmetric matrix

$$
\tilde{B}=\left[\begin{array}{ccc}
2 & 0 & -\frac{3}{2} \alpha \\
0 & 2 & 0 \\
-\frac{3}{2} \alpha & 0 & 4+\alpha^{2}
\end{array}\right]
$$

Since $\tilde{B}$ is positive definite for all $\alpha \in[-\sqrt{2}, \sqrt{2}]$ the Euler vector field is transverse to any level set of $\tilde{V}$ and hence any level set of $V$ for $\|x\|$ sufficiently small. $V\left(t, \Delta_{\lambda} x\right)$ is a monotone increasing function of $\lambda$ in a neighborhood of the origin. In this case, a bisection routine is used to calculate the required $\lambda$. Numerical calculation reveals that the level set value $C=1$ works well. Once the value of $\lambda$ has been computed which satisfies $V\left(t, \Delta_{\lambda} x\right)=1$, then we set $\tilde{\rho}(t, x)=1 / \lambda$ and $\bar{x}=\gamma(t, x)=\Delta_{\lambda} x$. The modified feedbacks are

$$
\begin{aligned}
\tilde{u}_{1}(t, x) & =\frac{1}{\lambda}\left(-\bar{x}_{1}+\bar{x}_{3} \cos t\right) \\
& =\frac{1}{\lambda}\left(-\lambda x_{1}+\lambda^{2} x_{3} \cos t\right) \\
& =-x_{1}+\lambda x_{3} \cos t \\
\tilde{u}_{2}(t, x) & =\frac{1}{\lambda}\left(-\bar{x}_{2}+\bar{x}_{3}^{2} \sin t\right) \\
& =\frac{1}{\lambda}\left(-\lambda x_{2}+\lambda^{4} x_{3}^{2} \sin t\right) \\
& =-x_{2}+\lambda^{3} x_{3}^{2} \sin t .
\end{aligned}
$$

Simulations comparing the performance of these feedbacks with the original smooth feedbacks are shown in Fig. 2. The $\rho$-exponential stabilizer returns the system to a small neighborhood of the origin much faster than the smooth controller from which it was derived. The Euclidean norm of control commands are shown in Fig. 3. The maximum effort expended by the $\rho$-exponentially stabilizing control law is slightly larger than that of the smooth controller.

Example 2: Consider a controller derived with Pomet's algorithm [47] for the system in (11). The reader is referred to [47] for the details on the algorithm. The open-loop periodic generator is chosen as $u_{2}(t)=\alpha(t, x)=x_{3}(t) \cos t$. The Lyapunov function defined with this preliminary input is $V(t, x)=1 / 2\left(x_{1}^{2}+\left(x_{2}-x_{3} \sin t\right)^{2}+x_{3}^{2}\right)$. The asymptotically stabilizing feedbacks are computed to be

$$
\begin{aligned}
& u_{1}=-x_{1}-x_{2}\left(x_{3}-\left(x_{2}-x_{3} \sin t\right) \sin t\right) \\
& u_{2}=x_{3} \cos t-x_{2}+x_{3} \sin t .
\end{aligned}
$$



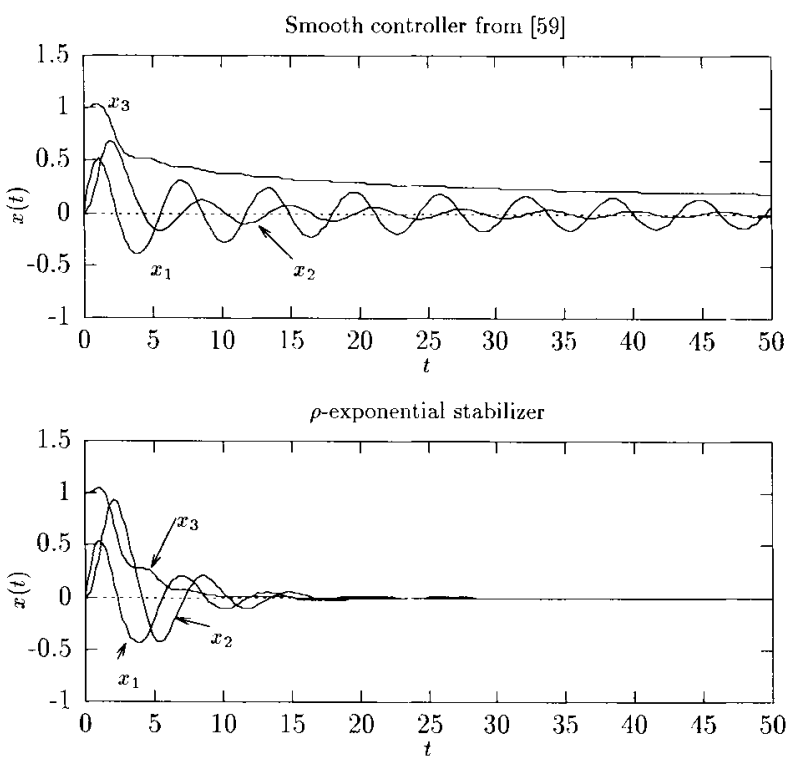

Fig. 2. (a) Comparison of system response with smooth feedback (b) and its modified version.

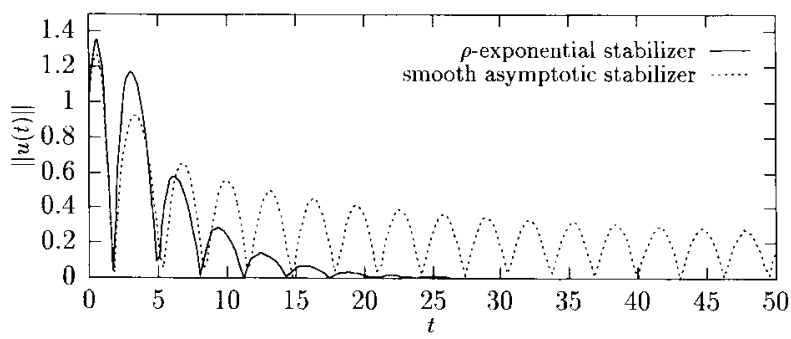

Fig. 3. Comparison of control efforts between the smooth and modified controllers.

The closed-loop system is globally asymptotically stable with this feedback although $\dot{V} \leq 0$ (Proposition 3 applies). The gradient of $V$ with respect to $X_{E}$ is a quadratic form

$$
L_{X_{E}} V=\left[\begin{array}{lll}
x_{1} & x_{2} & x_{3}
\end{array}\right]\left[\begin{array}{ccc}
1 & 0 & 0 \\
0 & 1 & -\frac{3}{2} \sin t \\
0 & -\frac{3}{2} \sin t & 2+2 \sin ^{2} t
\end{array}\right]\left[\begin{array}{l}
x_{1} \\
x_{2} \\
x_{3}
\end{array}\right] .
$$

This quadratic form is positive definite and implies that $X_{E}$ is transverse to the level sets of $V$. Note that since $V$ is quadratic, the transversality condition holds globally, i.e., any level set of $V$ may be chosen as the scaling set. A level set of $V(t, x)=0.5$ is chosen since the initial condition $x(0)=(0,0,1)$ is located on this set. Bisection is used to calculate $\lambda$ which satisfies $V\left(t, \Delta_{\lambda} x\right)=0.5$. The results of the simulations are shown in Figs. 4 and 5.

An important issue is the control effort used in stabilizing the system. Both the maximum control magnitude and the energy in the control signal are useful quantities to consider. The control magnitude will be limited by actuator constraints, and the amount of energy available to the controller will be dictated by the physical power source. It is straightforward to verify that

$$
\sup _{x \in U_{1}, t \in \mathbb{R}}|\tilde{u}(t, x)| \leq \sup _{x \in U_{2}, t \in \mathbb{R}}|u(t, x)|
$$
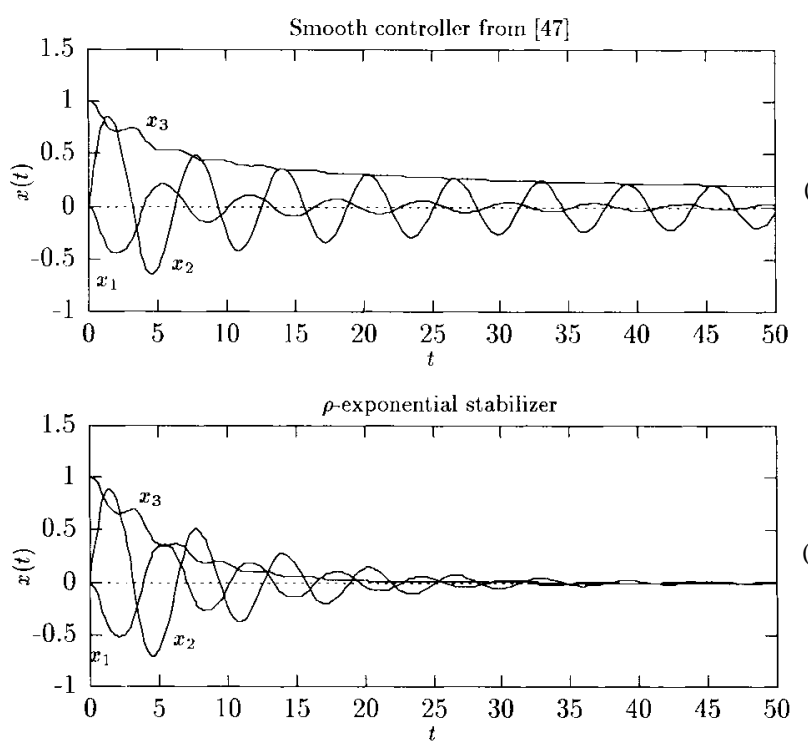

(b)

Fig. 4. (a) Comparison of systems responses with another smooth stabilizer and (b) its modified version.

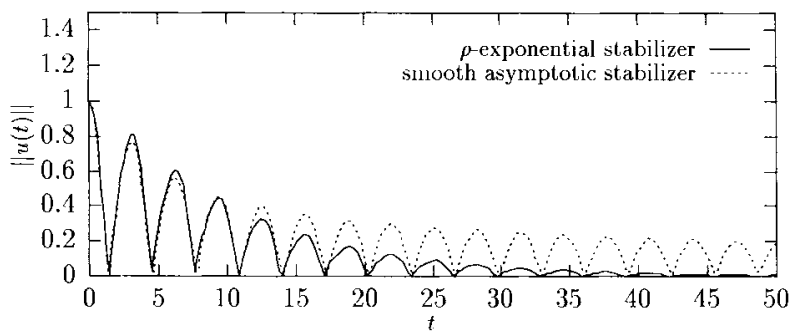

Fig. 5. Control effort comparison.

where $U_{1}=\cap_{t}\{x \mid V(t, x) \leq C\}$ and $U_{2}=\cup_{t}\{x \mid V(t, x) \leq$ $C\}$. Thus the control effort for the homogeneous feedbacks with initial conditions in $U_{1}$ will not exceed the control effort commanded by the original feedbacks with initial conditions in $U_{2}$. If $U_{1}$ is not much "smaller" than $U_{2}$, then the homogeneous feedbacks will $\rho$-exponentially stabilize the equilibrium point, for approximately the same set of initial conditions as the original controller, with no increase in maximum control magnitude.

Finally, since the homogeneous controllers have a Hölder bound of the form $\|u(t, x)\| \leq\|x\|^{\sigma}, \sigma \in(0,1)$, the energy in the control signal is guaranteed to be finite. In the examples above, the rate of convergence of the $x_{3}$ variable with the smooth controller is approximately $1 / \sqrt{t}$ for large $t$. Thus the smooth controllers in these examples expend an infinite amount of energy to return the system to the origin.

\section{EXPERIMENTAL RESULTS}

Feedbacks derived from Theorem 4 are applied to an experimental mobile robot. A picture of the robot, with two trailers, is shown in Fig. 6. The straight linkages are used to sense the configuration of the robot and do not affect its operation. The robot is configured so that it models the 


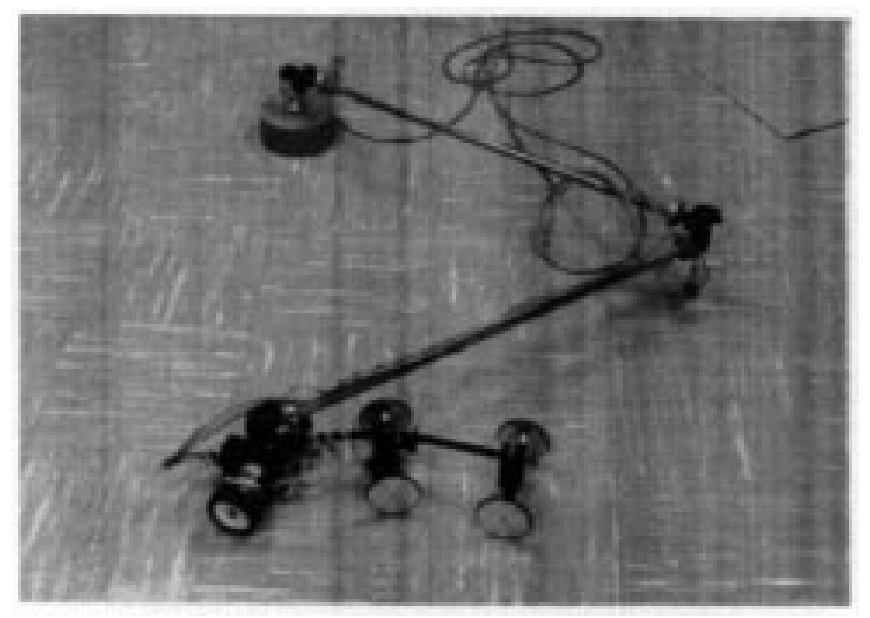

Fig. 6. Experimental mobile robot.

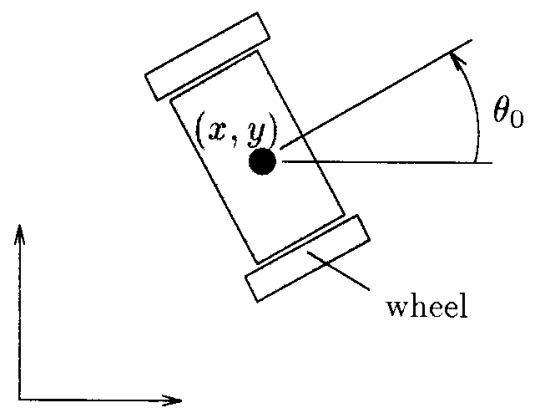

Fig. 7. Coordinate system for the robot.

"kinematic wheel"

$$
\begin{aligned}
& \dot{x}=\cos \theta \nu \\
& \dot{y}=\sin \theta \nu \\
& \dot{\theta}=\omega
\end{aligned}
$$

where the coordinates $(x, y, \theta)$ are used to describe the position and orientation of the robot (see Fig. 7). The control input $\nu$ is the forward velocity of the robot and $\omega$ its angular velocity. Forward and angular motion of the robot is achieved by changing the relative angular velocities of the wheels. Each wheel is driven by a stepper motor, and any desired wheel angular velocity is achieved by commanding the motors to turn the appropriate number of steps per second. Sensing the position and orientation is accomplished with a passive two degree-of-freedom linkage which is attached to the robot and a fixed base. More details on the experimental apparatus, results with other $\rho$-exponentially stabilizing controllers and additional trailers, and important controller design issues are related in our paper [35].

A preliminary coordinate change is performed before deriving the feedbacks. Define the new coordinates as

$$
\begin{aligned}
& z_{1}=\theta \\
& z_{2}=x \cos \theta+y \sin \theta \\
& z_{3}=x \sin \theta-y \cos \theta .
\end{aligned}
$$
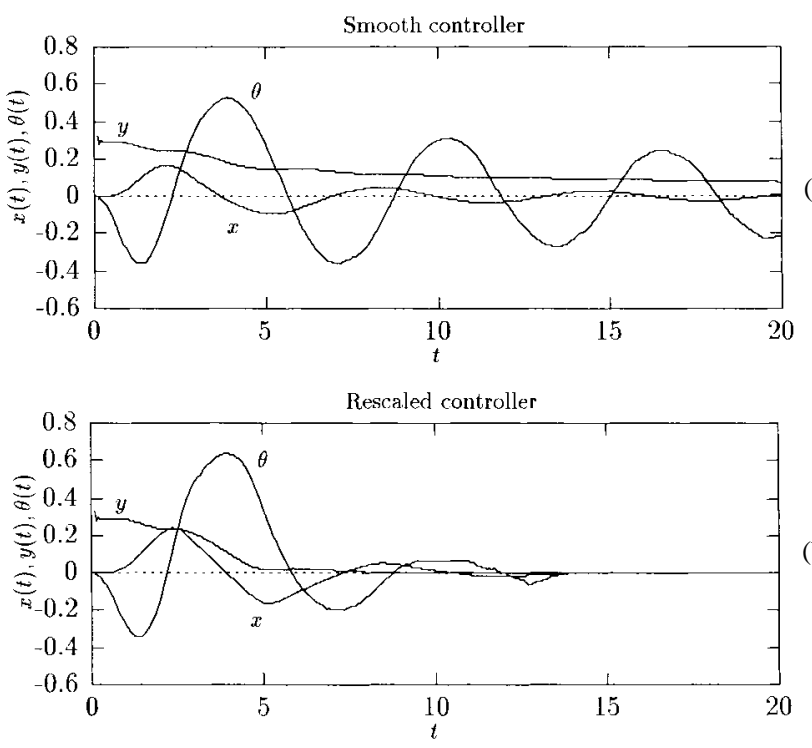

Fig. 8. Response of the experimental mobile robot with the smooth feedback and the $\rho$-exponential stabilizer.

The system equations transform to

$$
\begin{aligned}
& \dot{z}_{1}=\omega \\
& \dot{z}_{2}=\nu-z_{3} \omega \\
& \dot{z}_{3}=z_{2} \omega .
\end{aligned}
$$

It is simple to verify that the $\mathcal{F}$-approximation of the system in (17) is obtained by dropping the $z_{3} \omega$ term from $\dot{z}_{2}$. The input vector fields are $X_{1}(z)=\partial / \partial z_{1}+z_{2} \partial / \partial z_{3}$ and $X_{2}(z)=$ $\partial / \partial z_{2}$ and are homogeneous degree one with respect to the dilation $\Delta_{\lambda} z=\left(\lambda z_{1}, \lambda z_{2}, \lambda^{2} z_{3}\right)$. A smooth homogeneous norm is $\rho(z)=\left(z_{1}^{4}+z_{2}^{4}+z_{3}^{2}\right)^{\frac{1}{4}}$. This example is an instance where an initial transformation places the system into a form very close to the homogeneous approximation. Since the coordinate change in (16) is a global diffeomorphism, the feedbacks are defined over a large domain of the $(x, y, \theta)$ state space. Had we taken the homogeneous approximation directly from the original system (15), the resulting coordinate change is generally a local diffeomorphism. This would restrict the region of validity of the control law to the set where the coordinate change is well defined. In practice, it is always desirable to take advantage of these preliminary changes of coordinates if they can be found.

The approximate system is the system in (11). A locally stabilizing smooth feedback is given in (13). The response of the mobile robot with this feedback is in Fig. 8(a). The initial conditions are approximately $(x(0), y(0), \theta(0))=$ $(0 \mathrm{~m}, 0.3 \mathrm{~m}, 0 \mathrm{rad})$. The slow convergence rate is evident from this figure. In an effort to improve the convergence rate, the smooth control law is modified to the homogeneous control law as outlined in Example 1. The rescaling is performed in real-time during the experiment so the law cannot be written down explicitly. However, computing $\tilde{\rho}$ is simple since $V\left(t, \Delta_{\lambda} x\right)$ is a monotone increasing function of $\lambda$ in a neighborhood of the origin. A bisection search was employed to calculate $\tilde{\rho}$. This search was performed, along with the other control calculations, while a servo loop was running at $20 \mathrm{~Hz}$ 


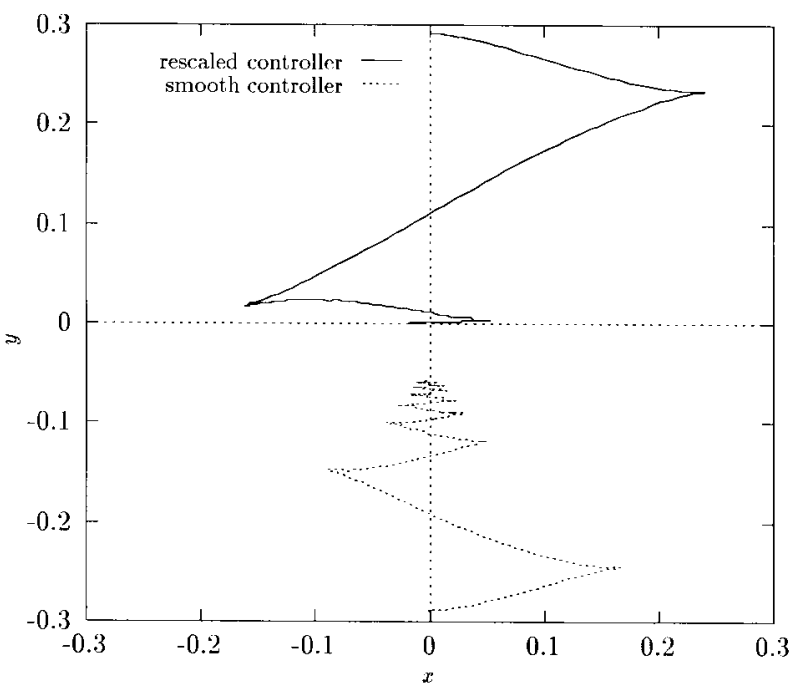

Fig. 9. Top view of mobile robot trajectories.

on a 486 PC. The response of the robot with the rescaled feedback is the second plot in Fig. 8. Note that although the transformed variables $z$ satisfy a bound of the form

$$
\rho(z(t)) \leq \beta \rho(z(0)) e^{-\alpha t}, \quad \text { some } \alpha, \beta>0
$$

the physical variables $x, y$, and $\theta$ satisfy the bound

$$
\|(x, y, \theta)(t)\| \leq M\|(x, y, \theta)(0)\|^{\frac{1}{2}} e^{-\alpha t} \quad \text { some } M>0 .
$$

The convergence to the origin has shown vast improvement: after fifteen seconds the robot has returned to its desired configuration with the homogeneous feedback, whereas the $y$ position of the robot is $8 \mathrm{~cm}$ from the desired position with the smooth feedback. Another useful plot is a "top view" of the robot trajectory. This is shown in Fig. 9 where $y(t)$ is plotted with respect to $x(t)$. The trajectory of the robot with the smooth controller has been reflected about the $x$ axis in this figure in order to keep the plots uncluttered. The velocities specified by the control laws are shown in Fig. 10. Each figure shows the "step rate" commanded to each stepper motor. In other words, the commanded velocity is approximated by sending a number of pulses to the motor so that the pulse-per-second value is close to the commanded velocity. The maximum control efforts are very close, although the homogeneous control law effort exceeds that of the smooth control law by about $20 \%$. The motors saturate at about 450 steps/s. Commands larger than this maximum step rate value are not tracked because the motor cannot apply sufficient torque.

\section{TORQue InPUTS AND DyNAMIC EXTENSION}

The mobile robot in the previous section is an example of a mechanical system in which a kinematic model is used for control design. That is, the velocity of the system is assumed to be a direct input which can be manipulated. In physical systems, however, actuators exert forces. It is desirable to extend the homogeneous $\rho$-exponentially stabilizing kinematic controllers to $\rho$-exponentially stabilizing controllers that command forces.
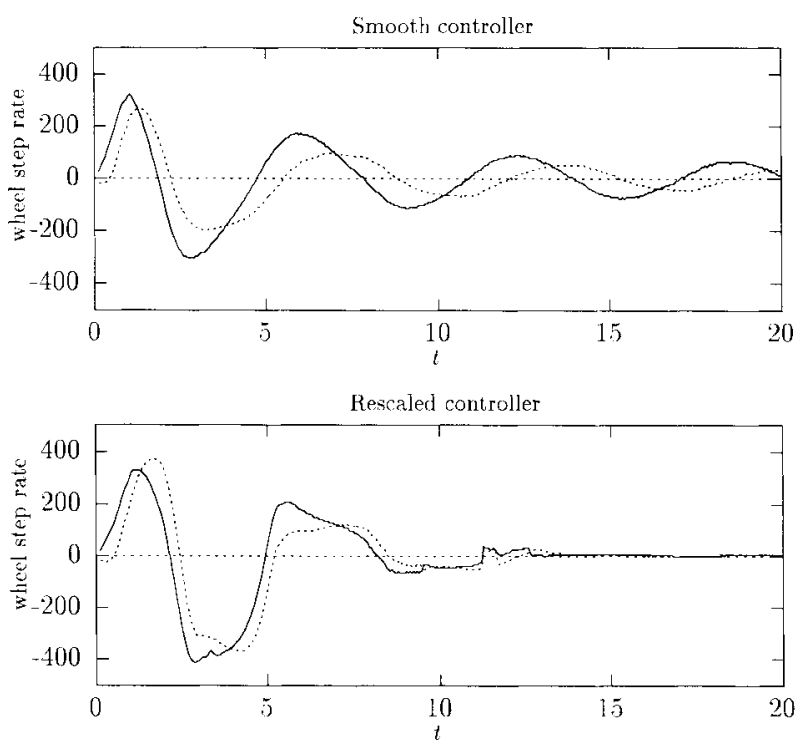

Fig. 10. Controller effort comparison for the experimental mobile robot.

The models we consider are very simple

$$
\begin{aligned}
& \dot{x}=X_{1}(x) u_{1}+\cdots+X_{m}(x) u_{m}, \quad x \in \mathbb{R}^{n} \\
& \dot{u}=v, \quad u, v \in \mathbb{R}^{m} .
\end{aligned}
$$

Equation (18) is termed the kinematic system. Equations (18) and (19) together represent the dynamic system. The kinematic system for mobile robots is derived from the Pfaffian constraints which describe the condition that the wheels roll but not slide. We model the dynamic portion of the system via a simple set of integrators. For many systems, more complicated dynamic behavior can be converted to this form using a state feedback control law [8].

The main result of this section gives a set of conditions under which a kinematic controller (i.e., one which assumes the velocities are the inputs) can be converted to a dynamic controller (one which uses the torques as the inputs) and still maintain $\rho$-exponential stability. Related results and an interesting application to attitude stabilization of a rigid body are given in [40].

For smooth controllers, extending kinematic controllers to dynamic controllers has been explored, for example, by Walsh and Bushnell [60]. However, due to the nondifferentiable nature of exponential stabilizers we consider here, the usual control Lyapunov approach does not directly apply and must be modified to verify that the extended controller is welldefined and continuous. The use of continuous functions is important in applications since discontinuous control inputs usually are smoothed by the control electronics and/or the system dynamics and hence cannot be applied in practice, possibly resulting in loss of exponential rate of convergence.

Proposition 4: Let the kinematic system in (18) satisfy the following conditions.

1) The vector fields $X_{i}$ are degree one with respect to a given dilation $\Delta_{\lambda}$.

2) The controls $u_{i}=\alpha_{i}(t, x), i=1, \cdots, m$ are uniformly asymptotically stabilizing feedbacks which are degree 
one in $x$ with respect to $\Delta_{\lambda}$, smooth on $\mathbb{R}^{n} \backslash\{0\}$, and time-periodic in $t$.

3) $\operatorname{rank}\left[X_{1}(0) \cdots X_{m}(0)\right]=m$.

Then, the feedback

$$
v_{i}=L_{\alpha X} \alpha_{i}+\frac{\partial \alpha_{i}}{\partial t}+k\left(\alpha_{i}-u_{i}\right), \quad i=1, \cdots, m
$$

globally exponentially stabilizes the dynamic system for $k>0$ sufficiently large.

The notation $\alpha X$ is used to denote the vector field $\sum_{i} \alpha_{i} X_{i}$. Controller (20) is continuous for all $(t, x, u)$ and smooth for all $x \neq 0$. Furthermore, the control law is homogeneous of degree one with respect to the extended dilation

$$
\tilde{\Delta}_{\lambda}(x, u)=\left(\lambda^{r_{1}} x_{1}, \cdots, \lambda^{r_{n}} x_{n}, \lambda u_{1}, \cdots, \lambda u_{m}\right) .
$$

Thus the closed-loop system remains degree zero with this feedback.

Proof: The closed-loop kinematic system is timeperiodic, degree zero, and asymptotically stable. This implies that there exists a time-periodic homogeneous Lyapunov function $V(t, x)$ such that $V(t, x)>0$ for all $x \neq 0$ and all $t$ which is strictly decreasing when $u=\alpha(t, x)$. This requires the converse Lyapunov theorem stated in Section IIIF. The Lyapunov function is chosen to be degree two with respect to $\Delta_{\lambda}$. Thus the following bounds exist:

$$
\begin{aligned}
& c_{1} \rho^{2}(x) \leq V(t, x) \leq c_{2} \rho^{2}(x) \\
& \left.\frac{d V}{d t}\right|_{\dot{x}=\alpha X}(t, x) \leq-c_{3} \rho^{2}(x)
\end{aligned}
$$

for some $c_{i}>0$ and where $\rho$ is a homogeneous norm with respect to $\Delta_{\lambda}$.

For the dynamic system with feedback (20) we use the following function:

$$
W(t, x, u)=V(t, x)+\frac{1}{2} \sum_{i=1}^{m}\left(\alpha_{i}(t, x)-u_{i}\right)^{2} .
$$

This function is positive definite on the extended phase space $(x, u)$ and thus is a Lyapunov function candidate. $W$ is degree two with respect to the extended dilation $\tilde{\Delta}$ defined in (21). Continuous partials of $W$ with respect to $x$ exist when $x \neq 0$, so in this case the derivative of (23) along the trajectories of the dynamic system with feedback (20) is

$$
\begin{aligned}
\dot{W}=\dot{V}+\sum_{i=1}^{m}( & \sum_{l=1}^{m}\left(\sum_{j=1}^{n} \frac{\partial \alpha_{i}}{\partial x_{j}} X_{l}^{(j)}\right) u_{l} \\
& \left.+\frac{\partial \alpha_{i}}{\partial t}-v_{i}\right)\left(\alpha_{i}-u_{i}\right), \quad x \neq 0
\end{aligned}
$$

where $X_{l}^{(j)}$ represents the $j^{\text {th }}$ component of the $l^{\text {th }}$ input vector field. Substituting the expression for $v_{i}$, the derivative may be expressed as

$$
\begin{gathered}
\dot{W}=\dot{V}+\sum_{i=1}^{m}\left(\sum_{l=1}^{m}\left(\sum_{j=1}^{n} \frac{\partial \alpha_{i}}{\partial x_{j}} X_{l}^{(j)}\right)\left(u_{l}-\alpha_{l}\right)\right. \\
\left.-k\left(\alpha_{i}-u_{i}\right)\right)\left(\alpha_{i}-u_{i}\right) \\
=\dot{V}+(\alpha-u)^{T}\left(-k I_{m}+Q(t, x)\right)(\alpha-u) .
\end{gathered}
$$

$I_{m}$ denotes the $m \times m$ identity matrix and $Q(t, x)$ is an $m \times m$ matrix with the $i j^{t h}$ component given by $[Q]_{i j}=$ $-1 / 2\left(L_{X_{i}} \alpha_{j}+L_{X_{j}} \alpha_{i}\right) \cdot L_{X_{i}} \alpha_{j}$ is a degree-zero function and is not necessarily defined at $x=0$.

Condition 3) in the proposition guarantees that no nontrivial trajectory of the closed-loop system is contained in the set $Z=\{(x, u): x=0, u \neq 0\}$. If a trajectory passes through the set $Z$ at time $t^{*}$, then $\frac{d W}{d t}\left(t^{*}\right)$ may not be defined; however, the continuity of $\frac{d W}{d t}\left(t^{*}+\epsilon\right)$ for $\epsilon>0$ implies that the upper right Dini derivative of $W(t)$ at $t^{*}$ is given by $D^{+} W\left(t^{*}\right)=\lim _{\epsilon \rightarrow 0^{+}} d W / d t\left(t^{*}+\epsilon\right)$. Substituting the original expression for $\dot{W}$ when $x \neq 0$ into the expression for $D^{+} W$ and noting that $\dot{V}$ is continuous in all arguments yields

$$
\begin{aligned}
D^{+} W\left(t^{*}\right) \\
=\lim _{\epsilon \rightarrow 0^{+}}\left[\frac{d V}{d t}+(\alpha-u)^{T}\left(-k I_{m}+Q\right)(\alpha-u)\right]_{t=t^{*}+\epsilon} \\
=\frac{d V}{d t}\left(t^{*}, x\left(t^{*}\right)\right)-k\left\|\alpha\left(t^{*}, x\left(t^{*}\right)\right)-u\left(t^{*}\right)\right\|^{2} \\
\quad+\lim _{\epsilon \rightarrow 0^{+}}\left[(\alpha-u)^{T} Q(\alpha-u)\right]_{t^{*}+\epsilon} \\
\leq \frac{d V}{d t}\left(t^{*}, x\left(t^{*}\right)\right)+(-k+q)\left\|\alpha\left(t^{*}, x\left(t^{*}\right)\right)-u\left(t^{*}\right)\right\|^{2}
\end{aligned}
$$

where $\|\cdot\|$ is the Euclidean norm and $q=$ $\sup _{t \in[0,2 \pi), x \neq 0}\|Q(t, x)\|$. The bound $q$ is well defined since $Q$ is degree zero and assumes all of its values when restricted to the homogeneous sphere $\{x: \rho(x)=1\}$. When $x \neq 0$, the expression for the derivative is continuous, so the Dini derivative reduces to the actual derivative. Thus the bound in (24) is valid for all $t, x$ and $u$

$$
D^{+} W(t) \leq d V / d t+(-k+q)\|\alpha-u\|^{2}, \quad \forall t, x, u .
$$

Substituting in $\dot{V}$ yields

$$
\begin{gathered}
D^{+} W \leq \frac{\partial V}{\partial t}+\sum_{k=1}^{m} \alpha_{k} L_{X_{k}} V+\sum_{k=1}^{m}\left(u_{k}-\alpha_{k}\right) L_{X_{k}} V \\
+(-k+q)\|\alpha-u\|^{2}, \quad \forall t, x, u .
\end{gathered}
$$

The first two terms on the right side of the inequality are the time derivative of $V$ along trajectories of the system when $u=\alpha(t, x)$ and may be bounded by $-c_{3} \rho^{2}(x)$ from (22). The third term to the right of the inequality may be bounded by $c_{4} \rho(x)\|u-\alpha\|$ for some $c_{4}>0$. Substituting these bounds into (25) yields

$$
\begin{aligned}
D^{+} W \leq & -c_{3} \rho^{2}(x)+c_{4} \rho(x)\|u-\alpha\|+(-k+q)\|\alpha-u\|^{2} \\
& =[\rho \quad\|u-\alpha\|]\left[\begin{array}{cc}
-c_{3} & \frac{1}{2} c_{4} \\
\frac{1}{2} c_{4} & -k+q
\end{array}\right]\left[\begin{array}{c}
\rho \\
\|u-\alpha\|
\end{array}\right] .
\end{aligned}
$$

This bound is negative definite when $k>k^{*} \doteq q+\frac{1}{4} \frac{c_{2}^{2}}{c_{1}}$. Furthermore, the bound is degree two with respect to the dilation $\tilde{\delta}_{\lambda}$ so $D^{+} W \leq-\tilde{k} W$ for some $\tilde{k}>0$ whenever $k>k^{*}$. The differential inequality from [29, Th. 1.4.1] implies $W(t) \leq W(0) e^{-\tilde{k} t}$. Hence, the system is asymptotically stable. Exponential stability follows from the fact that the closed-loop system is degree zero with respect to the extended dilation $\tilde{\delta}_{\lambda}$ defined in (21). 

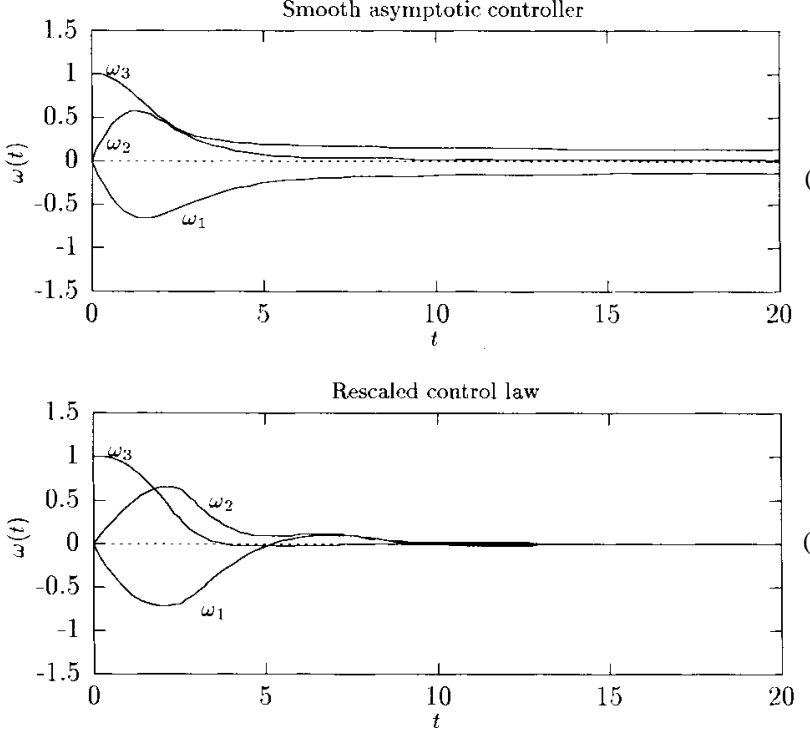

Fig. 11. (a) Response of the Euler equations with smooth feedback and (b) a $\rho$-exponential stabilizer.

The form of the control law shows that it can be regarded as a combined control law consisting of a feed-forward portion, which drives the system along the desired trajectory when $u=\alpha(t, x)$, and a feedback portion, which stabilizes the extended state-space equation.

\section{CONCLUSION}

Homogeneous feedbacks are an effective means to improve the convergence rate of driftless systems. The feedbacks are non-Lipschitz, and the states satisfy a modified definition of exponential stability. An algorithm is presented which gives conditions under which a smooth asymptotically stabilizing controller may be modified into a $\rho$-exponentially stabilizing feedback. The algorithm is applied to several examples and an experimental mobile robot.

The ideas in Theorem 4 also have a promising extension to systems with "drift" vector fields. However, the process of constructing the feedback is more ad hoc than the driftless case. For example, the Euler equation with two inputs can be used to demonstrate the scaling process on a system with drift vector field

$$
\begin{aligned}
& \dot{\omega}_{1}=u_{1} \\
& \dot{\omega}_{2}=u_{2} \\
& \dot{\omega}_{3}=\omega_{1} \omega_{2} .
\end{aligned}
$$

The system may be written as $\dot{\omega}=X_{0}+X_{1} u_{1}+X_{2} u_{2}$, where $X_{0}=\omega_{1} \omega_{2} \partial / \partial \omega_{3}, X_{1}=\partial / \partial \omega_{1}$ and $X_{2}=\partial / \partial \omega_{2}$. Defining the dilation $\Delta_{\lambda} \omega=\left(\lambda \omega_{1}, \lambda \omega_{2}, \lambda^{2} \omega_{3}\right)$, the drift vector field $X_{0}$ is degree zero and the input vector fields, $X_{1}$ and $X_{2}$, are degree one. For $\rho$-exponential stability, $u_{1}$ and $u_{2}$ should be degree-one functions with respect to this dilation since the closed-loop system will be degree zero with this choice. A smooth asymptotically stabilizing feedback is

$$
\begin{aligned}
& u_{1}(\omega)=-\omega_{1}-\omega_{3} \\
& u_{2}(\omega)=-\omega_{2}+\omega_{3}^{2} .
\end{aligned}
$$

Local asymptotic stability may be verified with the function

$$
V=\left(\omega_{1}+\omega_{3}\right)^{2}+\left(\omega_{2}-\omega_{3}^{2}\right)^{2}+\omega_{3}^{2} .
$$

The Euler vector field

$$
X_{E}=\omega_{1} \partial / \partial \omega_{1}+\omega_{2} \partial / \partial \omega_{2}+2 \omega_{3} \partial / \partial \omega_{3}
$$

corresponding to $\Delta_{\lambda}$ is locally transversal to the level sets of $V$. Thus it is possible to choose a level set of $V$ such that $\dot{V}<0$ on this set and $X_{E}$ is transversal to the set. By scaling $u_{1}$ and $u_{2}$ to degree-one functions on this level set, $\rho$ exponential stability can be proven using the same ideas found in the proof the Theorem 4. However, we rely on the fact that $X_{0}$ is degree zero in this example. The scaled feedbacks are smooth on $\mathbb{R}^{n} \backslash\{0\}$ but are not Lipschitz at the origin. Fig. 11 compares the performance of the smooth feedback in (26) versus its scaled version when the value of the level set used in defining the homogeneous feedback is $V(\omega)=1$.

\section{ACKNOWLEDGMENT}

The authors would like to thank L. Praly and the reviewers for their thoughtful comments.

\section{REFERENCES}

[1] M. Aicardi, G. Casalino, A. Bicchi, and A. Balestrino, "Closed loop steering of unicycle-like vehicles via Lyapunov techniques," IEEE Robotics Automat. Mag., vol. 2, no. 1, pp. 27-35, 1995.

[2] F. Ancona, "Homogeneous normal forms for vector fields with respect to an arbitrary dilation," Ph.D. dissertation, Univ. Colorado, 1993.

[3] A. Astolfi, "A unifying approach to the asymptotic stabilization of nonholonomic systems via discontinuous feedback," Automatic Control Lab, Swiss Federal Inst. Technol., ETH-Zurich, CH-8092 Zurich, Switzerland, Tech. Rep., Apr. 1994, draft.

[4] A. Bacciotti, Local Stabilizability of Nonlinear Control Systems, Series on Advances in Mathematics for Applies Sciences, vol. 8. World Scientific, 1992.

[5] G. Bastin and G. Campion, "Adaptive control of nonholonomic mechanical systems," in Proc. European Contr. Conf., 1991.

[6] A. Bellaïche, J. P. Laumond, and J. J. Risler, "Nilpotent infinitesimal approximation to a control Lie algebra," in Proc. IFAC Symp. Nonlinear Contr. Syst. Design (NOLCOS), 1992, pp. 174-181.

[7] A. Bloch and S. Drakunov, "Stabilization of a nonholonomic system via sliding modes," in Proc. IEEE Contr. Decision Conf., 1994, pp. 2961-2963.

[8] A. M. Bloch, M. Reyhanoglu, and N. H. McClamroch, "Control and stabilization of nonholonomic dynamic systems," IEEE Trans. Automat. Contr., vol. 37, pp. 1746-1757, 1992.

[9] R. W. Brockett, "Asymptotic stability and feedback stabilization," in Differential Geometric Control Theory, R. W. Brockett, R. S. Millman, and H. J. Sussman, Eds. Boston: Birkhauser, 1983, pp. 181-191.

[10] G. Casalino, M. Aicardi, A. Bicchi, and A. Balestrino, "Closed-loop steering for unicycle-like vehicles: A simple Lyapunov-like approach," in Proc. IFAC Symp. Robot Control (SYROCO), 1994, pp. 335-342.

[11] J.-M. Coron, "Global asymptotic stabilization for controllable systems without drift," Math. Contr., Signals, Syst., vol. 5, pp. 295-312, 1991.

[12] J.-M. Coron and J.-B. Pomet, "A remark on the design of time-varying stabilizing feedback laws for controllable systems without drift," in Proc. IFAC Symp. Nonlinear Contr. Syst. Design (NOLCOS), Bordeaux, France, June 1992, pp. 413-417.

[13] W. P. Dayawansa, C. F. Martin, and S. Samelson, "Asymptotic stabilization of a generic class of 3-dimensional homogeneous quadratic systems," Syst. Contr. Lett., vol. 24, no. 2, pp. 115-123, 1995.

[14] C. Canudas de Wit and O. J. Sørdalen, "Exponential stabilization of mobile robots with nonholonomic constraints," IEEE Trans. Automat. Contr., vol. 37, no. 11, pp. 1791-1797, 1992.

[15] O. Egeland and E. Berglund, "Control of an underwater vehicle with nonholonomic acceleration constraints," in Proc. IFAC Symp. Robot Contr. (SYROCO), 1994, pp. 959-964. 
[16] R. W. Goodman, Nilpotent Lie Groups: Structure and Applications to Analysis, Lecture Notes in Mathematics, no. 562. New York: SpringerVerlag, 1976.

[17] L. Gurvits, Personal communication, 1996.

[18] L. Gurvits and Z. X. Li, "Smooth time-periodic feedback solutions for nonholonomic motion planning," Courant Inst. Mathematical Sciences, New York, Tech. Rep. 1992 (preprint).

[19] W. Hahn, Stability of Motion. New York: Springer-Verlag, 1967.

[20] H. Hermes, "Nilpotent approximations of control systems and distributions," SIAM J. Contr. Optimization, vol. 24, no. 4, pp. 731-736, 1986.

[21] _ "Asymptotically stabilizing feedback controls and the nonlinear regulator problem," SIAM J. Contr. Optimization, vol. 29, no. 1, pp. 185-196, 1991.

[22] _ _ "Nilpotent and high-order approximations of vector field systems," SIAM Rev., vol. 33, no. 2, pp. 238-264, 1991.

[23] __ "Homogeneous feedback controls for homogeneous systems," Syst. Contr. Lett., vol. 24, pp. 7-11, 1995.

[24] A. Isidori, Nonlinear Control Systems, 2nd ed. New York: SpringerVerlag, 1989.

[25] M. Kawski, "Homogeneous feedback stabilization," in New Trends in Systems Theory, G. Conte, A. M. Perdon, and B. Wyman, Eds. Boston: Birkhäuser, 1990, pp. 464-471.

[26] _ "Homogeneous stabilizing feedback laws," Control-Theory and Advanced Technology, vol. 6, no. 4, pp. 497-516, 1990.

[27] _ "Geometric homogeneity and applications to stabilization," in Proc. IFAC Symp. Nonlinear Contr. Syst. Design (NOLCOS), 1995.

[28] I. V. Kolmanovsky and N. H. McClamroch, "Feedback stabilization of lower order nonholonomic systems in power form," Dept. Aerospace Engineering, Univ. Michigan, Tech. Rep., 1993 (preprint).

[29] V. Lakshmikantham and S. Leela, Differential and Integral Inequalities, vol. I. New York: Academic, 1969.

[30] N. E. Leonard, "Averaging and motion control of systems on Lie groups," Ph.D. dissertation, Univ. Maryland, 1994.

[31] B. M. Maschke and A. J. van der Schaft, "A Hamiltonian approach to stabilization of nonholonomic mechanical systems," in Proc. IEEE Contr. Decision Conf., 1994.

[32] R. T. M'Closkey, "Exponential stabilization of driftless nonlinear control systems," Ph.D. dissertation, California Inst. Technol., Pasadena, CA, 1994; available as memo CIT/CDS 95-003.

[33] R. T. M'Closkey and R. M. Murray, "Convergence rates for nonholonmic systems in power form," in Proc. Amer. Contr. Conf., 1993, pp. 2967-2972.

[34] _ - "Exponential covergence of nonholonomic systems: Some analysis tools," in Proc. IEEE Contr. Decision Conf., 1993, pp. 943-948.

[35] _ "Experiments in exponential stabilization of a mobile robot towing a trailer," in Proc. Amer. Contr. Conf., Baltimore, MD, 1994, pp. $988-993$.

[36] _ "Extending exponential stabilizers for nonholonomic systems from kinematic to dynamic controllers," in Proc. IFAC Symp. Robot Contr. (SYROCO), 1994, pp. 243-248.

[37] _ "Exponential stabilization of nonlinear driftless control systems via time-varying homogeneous feedback," in Proc. IEEE Contr. Decision Conf., Lake Buena Vista, FL, 1994, pp. 1317-1322.

[38] R. Montgomery, "Nonholonomic control and gauge theory," Nonholonomic Motion Planning, Z. Li and J. F. Canny, Eds. New York: Kluwer, 1993, pp. 343-378.

[39] P. Morin, "Sur la stabilixation par retour d'état instationnaire," Ph.D. dissertation, Thése de l'Ecole des Mines de Paris, 1996.

[40] P. Morin and C. Samson, "Time-varying exponential stabilization of the attitude of a rigid spacecraft with two controls," in Proc. 34th IEEE Conf. Decision Contr., 1995, pp. 3988-3993.

[41] R. M. Murray, Z. Li, and S. S. Sastry, A Mathematical Introduction to Robotic Manipulation. London: CRC, 1994

[42] R. M. Murray and R. T. M'Closkey, "Converting smooth, time-varying, asymptotic stabilizers for driftless systems to homogeneous, exponential stabilizers," in Proc. Third European Contr. Conf., 1995, pp. 2620-2925.

[43] R. M. Murray and S. S. Sastry, "Nonholonomic motion planning: Steering using sinusoids," IEEE Trans. Automat. Contr., vol. 38, no. 5m, pp. 700-716, 1993.

[44] R. M. Murray, G. Walsh, and S. S. Sastry, "Stabilization and tracking for nonholonomic systems using time-varying state feedback," in Proc. IFAC Symp. Nonlinear Contr. Syst. Design (NOLCOS), Bordeaux, France, 1992, pp. 182-187.

[45] H. Nijmeijer and A. J. van der Schaft, Nonlinear Dynamical Control Systems. New York: Springer-Verlag, 1990.
[46] W. Oelen, H. Berghuis, H. Nijmeijer, and C. Canudas de Wit, "Hybrid stabilizing control on a real mobile robot," IEEE Robotics Automat. Mag., vol. 2, pp. 16-23, 1995.

[47] J.-B. Pomet, "Explicit design of time-varying stabilizing control laws for a class of controllable systems without drift," Syst. Contr. Lett., vol. 18, no. 2, pp. $147-158,1992$.

[48] J.-B. Pomet and C. Samson, "Time-varying exponential stabilization of nonhlonomic systems in power form," INRIA, Tech. Rep. 2126, 1993.

[49] , "Time-varying exponential stabilization of nonhlonomic systems in power form," in Proc. IFAC Symp. Robust Contr. Design, 1994, pp. $447-452$.

[50] J.-B. Pomet, B. Thuillot, G. Bastin, and G. Campion, "A hybrid strategy for the feedback stabilization of nonholonomic mobile robots," Centre for Systems Engineering and Applied Mechanics, Universitè Catholique de Louvain, Belgium, Tech. Rep. 91.74, 1991.

[51] L. Praly, "Modification de l'échelle de temps en fonction de l'état," CAS École des Mines, France, Tech. Rep. 1995 (preprint).

[52] L. Rosier, "Homogeneous Lyapunov function for homogeneous continuous vector field," Syst. Contr. Lett., vol. 19, no. 6, pp. 467-473, 1992.

[53] C. Samson, "Velocity and torque feedback control of a nonholonomic cart," in Proc. Advanced Robot Contr., vol. 162. New York: SpringerVerlag, 1991.

[54] _ "Control of chained systems: Application to path following and time-varying point-stabilization of mobile robots," IEEE Trans. Automat. Contr., vol. 40, no. 1, pp. 64-77, 1995.

[55] E. Sontag, "Universal nonsingular controls," Syst. Contr. Lett., vol. 19 no. 3, pp. 221-224, 1992.

[56] "Feedback stabilization of nonlinear systems," in Mathematical Theory of Networks and Systems. Boston: Birkhauser, 1989.

[57] O. J. Sørdalen and O. Egeland, "Exponential stabilization of nonholonomic chained systems," IEEE Trans. Automat. Contr., vol. 40, no. 1, pp. 35-49, 1995 .

[58] O. J. Sørdalen, Y. Nakamura, and W. J. Chung, "Design of a nonholonomic manipulator," in Proc. IEEE Int. Conf. Robotics Automat., 1994, pp. $8-13$.

[59] A. R. Teel, R. M. Murray, and G. C. Walsh, "Non-holonomic control systems: from steering to stabilization with sinusoids," Int. J. Contr., vol. 62, no. 4, pp. 849-870, 1995.

[60] G. Walsh and L. Bushnell, "Stabilization of multiple input chained form control systems," in Proc. IEEE Contr. Decision Conf., 1993, pp. 959-964.

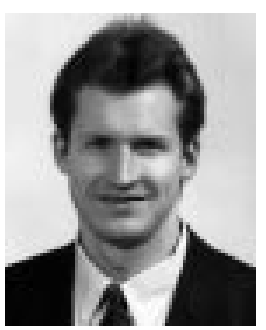

Robert T. M'Closkey (M'96) received the B.S. degree from the University of Windsor in 1987 and the M.S. and Ph.D. degrees from the California Institute of Technology, Pasadena, in 1988 and 1995, respectively, all in mechanical engineering.

He held a Post-Doctoral appointment in the Mechanical Engineering Department of the University of California, Berkeley, in 1995 and is currently an Assistant Professor in the Mechanical and Aerospace Engineering Department at the University of California, Los Angeles. His research interests include control system analysis and design tools for aircraft, microsensors, and nonlinear mechanical systems.

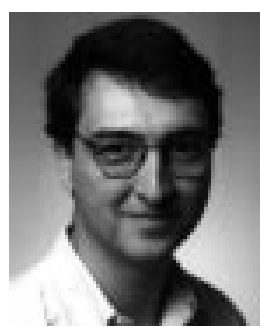

Richard M. Murray (S'85-M'90) received the B.S. degree in electrical engineering from California Institute of Technology, Pasadena, in 1985 and the M.S. and Ph.D. degrees in electrical engineering and computer sciences from the University of California, Berkeley, in 1988 and 1991, respectively.

He held a Post-Doctoral appointment at the University of California, Berkeley, in 1991 and is currently an Assistant Professor of Mechanical Engineering at the California Institute of Technology, Pasadena. His research interests include nonlinear control of mechanical systems with applications to aerospace vehicles and robotic locomotion, active control of fluids with applications to propulsion systems, and nonlinear dynamical systems theory. 